The Astrophysical Journal Supplement Series, 173:377-391, 2007 December

(C) 2007. The American Astronomical Society. All rights reserved. Printed in U.S.A.

\title{
ULTRAVIOLET, OPTICAL, AND INFRARED CONSTRAINTS ON MODELS OF STELLAR POPULATIONS AND DUST ATTENUATION
}

\author{
Benjamin D. Johnson, ${ }^{1}$ David Schiminovich, ${ }^{1}$ Mark Seibert, ${ }^{2}$ Marie Treyer, ${ }^{3,4}$ D. Christopher Martin, ${ }^{4}$ \\ Tom A. Barlow, ${ }^{4}$ Karl Forster, ${ }^{4}$ Peter G. Friedman, ${ }^{4}$ Patrick Morrissey, ${ }^{4}$ Susan G. Neff, ${ }^{5}$ \\ Todd Small, ${ }^{4}$ Ted K. Wyder, ${ }^{4}$ Luciana Bianchi, ${ }^{6}$ José Donas, $^{3}$ Timothy M. Heckman, ${ }^{7}$ \\ Young-Wook Lee, ${ }^{8}$ Barry F. Madore, ${ }^{2}$ Bruno Milliard, ${ }^{3}$ R. Michael Rich, ${ }^{9}$ \\ Alex S. Szalay, ${ }^{7}$ Barry Y. Welsh, ${ }^{10}$ and Sukyoung K. Y Y ${ }^{8}$ \\ Received 2007 February 26; accepted 2007 August 30
}

\begin{abstract}
The color of galaxies is a fundamental property, easily measured, that constrains models of galaxies and their evolution. Dust attenuation and star formation history (SFH) are the dominant factors affecting the color of galaxies. Here we explore the empirical relation between $\mathrm{SFH}$, attenuation, and color for a wide range of galaxies, including early types. These galaxies have been observed by GALEX, SDSS, and Spitzer, allowing the construction of measures of dust attenuation from the ratio of infrared (IR) to ultraviolet (UV) flux and measures of SFH from the strength of the $4000 \AA$ break. The empirical relation between these three quantities is compared to models that separately predict the effects of dust and SFH on color. This comparison demonstrates the quantitative consistency of these simple models with the data and hints at the power of multiwavelength data for constraining these models. The UV color is a strong constraint; we find that a Milky Way extinction curve is disfavored, and that the UV emission of galaxies with large $4000 \AA$ break strengths is likely to arise from evolved populations. We perform fits to the relation between SFH, attenuation, and color. This relation links the production of starlight and its absorption by dust to the subsequent reemission of the absorbed light in the IR. Galaxy models that self-consistently treat dust absorption and emission as well as stellar populations will need to reproduce these fitted relations in the low-redshift universe.
\end{abstract}

Subject headings: dust, extinction — galaxies: evolution — galaxies: fundamental parameters infrared: galaxies — ultraviolet: galaxies

\section{INTRODUCTION}

Since the work of Tinsley (1968) the location of galaxies in color-color and color-magnitude diagrams has proven to be an important indicator of the stellar content of galaxies, just as the location of stars in these same diagrams indicates their stellar spectral type. This stellar content is in turn used to constrain the star formation histories (SFHs) of galaxies, which is an important step toward understanding the diversity of galaxies observed in the universe today. Color-color diagrams are still used at high redshift (and low), where in addition to the stellar populations and dust attenuation, the redshift is estimated (Schweizer \& Seitzer 1992; Mobasher et al. 2004; Wuyts et al. 2007).

To obtain strong constraints on the constituent stellar populations with color-color diagrams, it is important to know the properties of the dust attenuation in galaxies. Selective attenuation can

\footnotetext{
${ }^{1}$ Department of Astronomy, Columbia University, New York, NY 10027.

2 Observatories of the Carnegie Institution of Washington, 813 Santa Barbara Street, Pasadena, CA 91101.

3 Laboratoire d'Astrophysique de Marseille, BP 8, Traverse du Siphon, 13376 Marseille Cedex 12, France.

${ }^{4}$ California Institute of Technology, 1200 East California Boulevard, MC 405-47, Pasadena, CA 91125.

5 Laboratory for Astronomy and Solar Physics, NASA Goddard Space Flight Center, Greenbelt, MD 20771.

${ }^{6}$ Center for Astrophysical Sciences, The Johns Hopkins University, 3400 North Charles Street, Baltimore, MD 21218.

${ }^{7}$ Department of Physics and Astronomy, The Johns Hopkins University, Homewood Campus, Baltimore, MD 21218.

${ }^{8}$ Center for Space Astrophysics, Yonsei University, Seoul 120-749, Korea.

9 Department of Physics and Astronomy, University of California, Los Angeles, CA 90095.

${ }^{10}$ Space Sciences Laboratory, University of California at Berkeley, 601 Campbell Hall, Berkeley, CA 94720.
}

cause a large amount of reddening that can mask the differences in color due to differences in stellar populations. Such attenuation is often treated as a reddening vector in color-color space (e.g., Kauffmann et al. 2003b). The direction of the vector is given by the shape of the attenuation curve (often assumed to be that of Calzetti et al. 1994), while the length of the vector is inferred indirectly. Without independent knowledge of the length of the reddening vector, the power of color-color diagrams to probe the constituent stellar populations of galaxies is reduced.

The proper treatment of dust attenuation is of paramount importance when considering the constraints imposed by the colormagnitude diagram (CMD) on theories of galaxy formation (e.g., Noeske et al. 2007; Labbe et al. 2007). This is especially true since there is a strong correlation of attenuation with luminosity (Wang \& Heckman 1996) and stellar mass (e.g., Salim et al. 2007). These correlations can induce or alter trends between the derived SFH and stellar mass of galaxies. Similarly, dust attenuation also affects comparisons between the predictions of semianalytic modeling and observations of galaxies at any wavelength.

With the Galaxy Evolution Explorer (GALEX) and Spitzer Space Telescope it is now possible to obtain ultraviolet (UV) and infrared (IR) fluxes for large samples of galaxies. The combination of the UV and IR flux constrains the amount of dust attenuation in galaxies (i.e., the length of the reddening vector). The UV is due to emission from young stars that is not attenuated by dust, while the IR measures the emission from young stars that has been absorbed by dust and reradiated. With this independent knowledge of the dust attenuation, which significantly affects the observed color of galaxies, these colors can be used to more sensitively probe the stellar content of galaxies and test models of galaxy formation. New models of galaxy spectra are being developed and improved that treat dust attenuation, dust emission, and 
TABLE 1

OBSERVATIONS

\begin{tabular}{cccccc}
\hline \hline Field Name & $\begin{array}{c}\text { Size } \\
\left(\mathrm{deg}^{2}\right)\end{array}$ & $N_{\text {obs }}{ }^{\mathrm{a}}$ & $N_{\text {det }}{ }^{\mathrm{b}} f, n<25$ & $N_{\text {det }}{ }^{\mathrm{b}} m_{24}<19.5$ & $N_{\text {smpl }}{ }^{\mathrm{c}}$ \\
\hline Lockman Hole …………... & $\sim 9$ & 872 & 792 & 819 & 721 \\
FLS ................................ & $\sim 3$ & 186 & 147 & 158 & 118 \\
\hline
\end{tabular}

Note.-The median redshift in both fields is $z=0.1$.

${ }^{\mathrm{a}}$ The number of SDSS/MPA galaxies that are observed at all $f$ through $24 \mu \mathrm{m}$ wavelengths ( $(2.2)$.

${ }^{b}$ The number of observed SDSS/MPA galaxies with fluxes brighter than the noted AB magnitude.

c The number of observed SDSS/MPA galaxies that pass the flux, redshift, and size cuts given in $\S 2.4$.

stellar populations in a consistent manner, building from the work of Charlot \& Fall (2000). These UV through IR models (e.g., Silva et al. 1998; Jonsson 2006) promise to improve the treatment of attenuation and sharpen the constraints imposed by UV and IR observations on theories and semianalytic models of galaxy formation.

In Johnson et al. (2006) we presented the relation between broadband UV through near-IR colors, dust attenuation (as measured by the infrared excess [IRX]), and SFH (as measured by the $4000 \AA$ break) for a sample of galaxies observed with GALEX, the Sloan Digital Sky Survey (SDSS), and Spitzer. Here we present this same relation, with deeper UV data, for a larger sample of galaxies and a greater variety of broadband colors. We show that this relation serves as a useful diagnostic of galaxy properties and an empirical constraint on models of dust attenuation and star formation (SF). We compare our results to models of galaxy spectra (Bruzual \& Charlot 2003) and dust attenuation (Witt \& Gordon 2000). In addition, we consider where dwarf galaxies and IR- and UV-luminous galaxies fall in this diagram. This comparison of special classes of galaxies to a more representative sample places them in context and aids the interpretation of the relation.

We present parameterized fits to this relation between color, the $4000 \AA$ break strength, and IRX. These fits may be used to determine the dust attenuation in galaxies where only optical spectra are available, even in the absence of measurable $\mathrm{H} \alpha$ and $\mathrm{H} \beta$ lines. In addition, these relations provide an empirical guide for self-consistent models of the UV through IR spectra of galaxies.

\section{DATA}

Our primary sample is selected from the SDSS main galaxy spectroscopic sample (Strauss et al. 2002). These galaxies have been imaged in five optical bands (ugriz) and observed spectroscopically from 3800 to $9000 \AA$ (observed frame) in a $3^{\prime \prime}$ diameter aperture as part of the SDSS.

In this paper we consider a subset of the SDSS spectroscopic galaxy sample that has been observed by both GALEX and Spitzer. These galaxies are located in two different contiguous regions, the Lockman Hole and the Spitzer extragalactic First Look Survey (FLS). Details of the observations for each of these fields are given in Table 1. The Spitzer observations include four-band Infrared Array Camera (IRAC; Fazio et al. 2004) imaging (3.6, $4.5,5.8$, and $8 \mu \mathrm{m}$ ), as well as 24 and $70 \mu \mathrm{m}$ Multiband Imaging Photometer for Spitzer (MIPS; Rieke et al. 2004) imaging. The GALEX observations include imaging at $1517 \AA$ (far-UV, $f$ ) and $2267 \AA$ (near-UV, $n$ ). All quoted magnitudes are on the AB system and have been corrected for foreground extinction (which is especially low in the case of the Lockman Hole) according to the maps of Schlegel et al. (1998).

\subsection{Optical Data and Derived Parameters: SDSS}

A description of the SDSS optical reductions is given in Stoughton et al. (2002) and Abazajian et al. (2004). Throughout this study we use the Petrosian magnitudes supplied by the SDSS Data Release 4 (DR4), as given in the photometric catalogs provided by the MPA/JHU group for SDSS studies. ${ }^{11}$ Additional measurements of emission-line strengths and spectroscopic index values have been made by the MPA/JHU group using specialpurpose code to improve continuum subtraction and are available from the MPA/JHU Web site. We have removed duplicate galaxies in these catalogs.

Additional parameters including star formation rates (SFRs) and stellar masses have also been derived and made publicly available for many of these galaxies by Kauffmann et al. (2003b) and Brinchmann et al. (2004). The stellar mass estimates are obtained by Kauffmann et al. (2003b) from fits of a suite of stellar population synthesis models to observed spectral indices (to obtain the dust-free mass-to-light ratio) and colors (to estimate the attenuation). These stellar masses are consistent with stellar masses derived using a simple magnitude and color prescription as in Bell $\&$ de Jong (2001) that are in turn consistent with limits from dynamical masses. The gas-phase metallicities of many of the sample galaxies have been determined by Tremonti et al. (2004) from emission-line fluxes. These metallicities are only available for galaxies with significant emission lines that are not classified as AGNs (see Kauffmann et al. [2003a] for the AGN classification criteria) and are only defined within the SDSS aperture.

We only consider galaxies for which a mass has been determined by Kauffmann et al. (2003b; hereafter SDSS/MPA galaxies). Readers are encouraged to see Kauffmann et al. (2003b) for a description of the optical selection. In total we consider 872 SDSS/MPA galaxies in the Lockman Hole that have been observed from the far-UV through $24 \mu \mathrm{m}$. Of these galaxies, 866 have also been observed at $70 \mu \mathrm{m}$. In the FLS field we consider an additional 186 galaxies observed in the UV (with sufficient UV effective exposure time; $\S 2.2$ ) through $24 \mu \mathrm{m}$.

\subsection{IR and UV Data}

The Lockman Hole field has been observed by GALEX in 26 $0.6^{\circ}$ radius circular tiles. The effective exposure times of these tiles are $\sim 10-15 \mathrm{ks}$ in $f$ and $\sim 10-60 \mathrm{ks}$ in $n$. In the FLS field the effective exposure times are highly variable from tile to tile, and we require exposure times $>5 \mathrm{ks}$ in both $f$ and $n$. The UV photometry is taken from the pipeline-produced catalogs provided by the GALEX science team (Morrissey \& Seibert 2007). For each SDSS/MPA galaxy we search the catalogs for objects within $3^{\prime \prime}$ of the SDSS position, choosing the closest object in the rare case of multiple matches. Since the GALEX fields overlap, some

${ }^{11}$ Available at http://www.mpa-garching.mpg.de/SDSS/. 
objects appear in two separate fields. We choose the tile with the longer effective exposure time (that takes into account sensitivity variations in the detector). We also exclude objects $>0.55^{\circ}$ from the GALEX field center, as the incidence of artifacts is much higher here. The UV photometry, the principal UV data used in this study, is computed in elliptical Kron apertures using a version of SExtractor (Bertin \& Arnouts 1996) modified to operate on lowbackground images.

The IR data are from publicly available Spitzer imaging. In the Lockman Hole we use the reduced images provided by the Spitzer Wide-Area Infrared Extragalactic Survey (SWIRE; Lonsdale et al. 2003) team. ${ }^{12}$ In the FLS field we use the post-basic calibrated data (post-BCD) data provided by the Spitzer Science Center. ${ }^{13}$ We have checked the consistency of these data sets by comparing the post-BCD Lockman Hole mosaics to the SWIRE-processed data in this field; the photometry is consistent to better than $0.1 \mathrm{mag}$ at the flux levels we consider, except at $70 \mu \mathrm{m}$ (which we do not consider for the FLS field) and at the faintest $24 \mu \mathrm{m}$ fluxes where the (random) differences rise to $\sigma \sim 0.5 \mathrm{mag}$ at $m_{24}=19 \mathrm{mag}$. We have performed aperture photometry in the Spitzer 3.6-8 $\mu \mathrm{m}$ IRAC images and $24 \mu \mathrm{m}$ MIPS images at the location of each of the SDSS/MPA galaxies, using a $7^{\prime \prime}$ radius aperture $\left(12^{\prime \prime}\right.$ at $24 \mu \mathrm{m})$. The IRAC fluxes are then aperture-corrected to $12.2^{\prime \prime}$, the radius used for IRAC standard star flux measurements. At $24 \mu \mathrm{m}$ we attempt to calculate total fluxes by applying an aperture correction to a very large radius $(>35)$ derived from the brightest $24 \mu \mathrm{m}$ sources. We have checked that the $7^{\prime \prime}$ radius aperture (12" at $24 \mu \mathrm{m}$ ) does not induce significant aperture effects by comparing to fluxes in larger radius apertures as a function of $r$-band Petrosian radius; no significant trend is seen for the galaxies of our final sample. At $70 \mu \mathrm{m}$ our sources are unresolved, and following Gordon et al. (2007) we apply an aperture correction of 2.07 to fluxes obtained with a $16^{\prime \prime}$ radius aperture with $18^{\prime \prime}-39^{\prime \prime}$ sky annulus. Systematic errors in IR flux due to calibration uncertainty, aperture corrections, and the resolved nature of many of the sources at wavelengths less than $70 \mu \mathrm{m}$ amount to $\sim 30 \%$.

\section{3. $\mathrm{K}$-Corrections and Luminosities}

The resulting UV through $3.6 \mu \mathrm{m}$ magnitudes are $K$-corrected to $z=0.1$, the median redshift of the sample (e.g., ${ }^{0.1} u,{ }^{0.1} g$, etc.), using the method of Blanton \& Roweis (2007). The generalized UV luminosities are then calculated as $L_{\mathrm{UV}}=4 \pi D_{L}^{2} f_{\lambda} \lambda_{f}$, where $D_{L}$ is the luminosity distance ${ }^{14}$ and $f_{\lambda}$ is the $K$-corrected flux density per unit wavelength in the ${ }^{0.1} f$ band, with effective wavelength $\lambda_{f}=1390 \AA$ A . At longer wavelengths dust emission becomes more important than stellar emission, and we use a different method to " $K$-correct" the data: we choose the best-fitting redshifted Dale et al. (2001) model IR spectral energy distribution (SED), on the basis of the observed 8-24 $\mu \mathrm{m}$ flux ratio, after correction for stellar emission at 8 and $24 \mu \mathrm{m}$ determined from the $3.6 \mu \mathrm{m}$ flux following Helou et al. (2004) and Dale et al. (2005). This best-fit SED is then normalized using the measured $24 \mu \mathrm{m}$ flux, and the integrated far-IR $(8-1000 \mu \mathrm{m})$ dust luminosities $\left(L_{\text {dust }}\right)$ are derived. Note that the different Dale et al. (2001) SEDs have $L_{24 \mu \mathrm{m}} / L_{\text {dust }}$ ratios that are different by a factor of up to 5 . We have checked that our results would not change significantly if we used the model SEDs of Devriendt et al. (1999; see Papovich \& Bell

\footnotetext{
${ }^{12} \mathrm{See}$ http://swire.ipac.caltech.edu/swire/astronomers.html for a description of the SWIRE image processing.

${ }_{13} \mathrm{See}$ http://ssc.spitzer.caltech.edu/postbcd/ for a description of post-BCD processing.

${ }_{14}$ Throughout this study we assume a concordance cosmology: $\Omega_{m}=0.3$, $\Omega_{\Lambda}=0.7, H_{0}=70 \mathrm{~km} \mathrm{~s}^{-1} \mathrm{Mpc}^{-1}$.
}

[2002] for a detailed discussion of predicting IR luminosities from Spitzer data).

In Figure 1 we show the far-IR flux ratios for the sample galaxies, compared to the models of Dale et al. (2001) and data from the Spitzer Infrared Nearby Galaxy Survey (SINGS) sample of galaxies (Kennicutt et al. 2003; Dale et al. 2005). Note that the $24 \mu \mathrm{m} / 70 \mu \mathrm{m}$ color does not vary as much as the $8 \mu \mathrm{m} / 24 \mu \mathrm{m}$ color for these models and is not monotonic. The $24 \mu \mathrm{m} / 70 \mu \mathrm{m}$ ratio for the sample galaxies, and for the SINGS galaxies, show a larger range of $L_{\nu}(24 \mu \mathrm{m}) / L_{\nu}(70 \mu \mathrm{m})$ than the Dale et al. (2001) models. Many of the galaxies in our sample are bluer in the $8 \mu \mathrm{m} / 24 \mu \mathrm{m}$ color than any of the Dale et al. (2001) models at their redshift, likely due to incomplete treatment in these models of PAH emission, which is assumed to be constant in spectral shape (see Smith et al. [2007] for evidence of changes in the relative strength of PAH features). In general the scatter observed in this diagram suggests that more complicated and multiparameter models may be necessary (Smith et al. 2007; Draine \& Li 2007). The use of such models with SDSS/MPA galaxies will be explored in future work. Some care must be taken in interpreting the total dust luminosities we derive, since these rely on models that do not appear to match existing data to high accuracy.

\subsection{Samples and Limits}

Our primary sample then consists of these galaxies with $m_{24}<$ $19.5(\mathrm{AB})$ and $f$ and $n<25.0(\mathrm{AB})$. It has been found that systematic errors in the $n$-band photometry due to source blending can occur at $n>23$; only 45 of our galaxies are at these magnitudes. The SDSS spectroscopic selection, from which our sample is drawn, also requires $r<17.7$ (AB). In the Lockman Hole, 80 of the 872 multiwavelength-observed SDSS/MPA galaxies fail the UV cut, and 53 galaxies fail the $24 \mu \mathrm{m}$ cut, of which 12 also fail the UV cut. We further require $z>0.02$ (8 galaxies removed) and an $r$-band Petrosian radius $\left[R_{\mathrm{P}}(r)\right]$ of less than $11^{\prime \prime}$ (an additional 23 galaxies removed) to avoid large galaxies which are difficult to accurately photometer. This leaves a total of 721 SDSS/MPA galaxies out of 872 observed at all wavelengths ( $f$ through $24 \mu \mathrm{m}$ ) in the Lockman Hole (82\%). The galaxies that are missed due to the UV and IR flux cuts are primarily red-sequence or early-type galaxies (of which $\sim 70 \%$ are detected), although several dwarf galaxies are also missed. The size cuts primarily remove blue dwarf galaxies, which tend to be at low redshift in the SDSS sample, although a number of dwarfs remain. In the FLS these same limits yield 118 galaxies detected at all wavelengths out of 186 observed $(\sim 63 \%)$. The lower detection rate in the FLS can be explained by the different UV exposure time distribution and the variation of the redshift distribution between the FLS and Lockman Hole fields (which both have median redshift $z=0.1$ ).

\subsection{Classes of Galaxies}

We define several special classes of galaxies to be used throughout this study; these follow common definitions in the literature and are nonexclusive.

1. Dwarfs, with $\log M_{*}<9$, where $M_{*}$ in solar units is from Kauffmann et al. (2003b).

2. Luminous infrared galaxies (LIRGs), with $L_{\text {dust }}>10^{11} L_{\odot}$.

3. Ultraviolet luminous galaxies (UVLGs), with $\nu L_{\nu}=L_{\mathrm{UV}}>$ $10^{10} L_{\odot}$. Note that this definition does not include structural parameters (e.g., compactness) which appear necessary in identifying galaxies that may serve as low-redshift analogs for Lyman break galaxies (Heckman et al. 2005).

We also consider the properties of galaxies that have been observed both as part of the SINGS program (Kennicutt et al. 2003) 


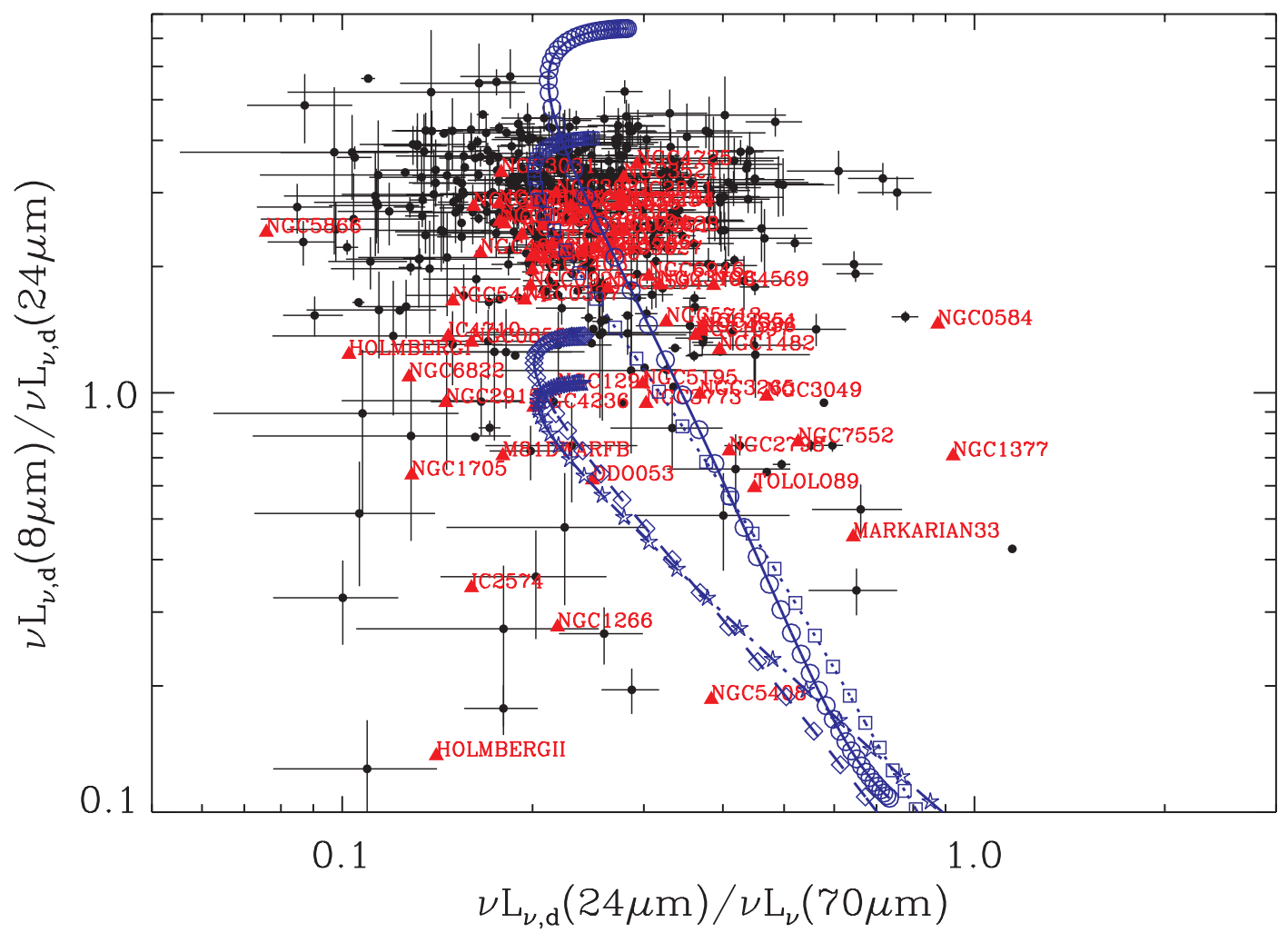

FIG. 1.-Far-IR color-color diagram for the sample with $70 \mu \mathrm{m} \mathrm{AB}$ magnitude $m_{70} \mu \mathrm{m}<1$. Also shown are integrated values from Dale et al. (2005) SINGS galaxies (red triangles) and the models of Dale et al. (2001; blue line, diamonds). The Dale et al. (2001) models are shown for redshifts of $z=0.0$ (open circles), 0.1 (squares), 0.2 (diamonds), and 0.3 (stars). The median redshift of the sample is $z=0.1$. Error bars show the photometric measurement errors.

and by GALEX as part of the Nearby Galaxy Survey (NGS; Gil de Paz et al. 2007). The integrated fluxes of these nearby, resolved galaxies are given by Dale et al. (2007). The 65 SINGS galaxies that we consider have publicly available drift-scan spectroscopy as well (Moustakas \& Kennicutt 2006), from which we have calculated a $4000 \AA$ break measure, $D_{n}(4000)$, for comparison with the SDSS $D_{n}(4000)$ values (see $\left.\S 3.1\right)$.

\section{THE GLOBAL PROPERTIES OF GALAXIES IN THE UV AND IR}

In Johnson et al. (2006) we introduced an observational diagnostic of galaxy properties that relates the SFH and attenuation of a galaxy to its color. The SFH and attenuation are the primary drivers of galaxy color (although metallicity can play a significant role, especially in older galaxies). A combination of IR and UV observations provides a measurement of obscured and unobscured SF and is thus ideal for measuring SFR and attenuation. A detailed exploration of this relation is given in $\S 4$. Here we investigate the location of nearby resolved galaxies, special classes of galaxies, and the sample of all detected galaxies in the CMD and the relation between color, $\mathrm{SFH}$, and attenuation. These additional diagnostic relations are the $\mathrm{CMD}$ and the relation between attenuation and SFR. The locations of the special classes of galaxies $(\S 2.5)$ both places them in the context of the larger, more general galaxy distribution and informs the interpretation of the relations. The measures of SFH and dust attenuation that we use are the strength of the $4000 \AA$ break and the ratio of IR to UV luminosities and are described here.

\section{1. $D_{n}(4000)$}

As a measure of SFH we use the definition of the $4000 \AA$ break strength given in Balogh et al. [1999; $\left.D_{n}(4000)\right]$, since it is mea- surable at high signal-to-noise ratio $(\mathrm{S} / \mathrm{N})$ for the galaxies that we consider (i.e., both red- and blue-sequence galaxies) and is nearly insensitive to dust reddening (MacArthur 2005). The $4000 \AA$ break is similar to a measure of the ratio of the SFR averaged over $\sim 10^{8.5} \mathrm{yr}$ to the SFR averaged over $>10^{9} \mathrm{yr}$ but is subject to strong variations with metallicity, especially at larger break strengths. In $\S 4$ we explore the behavior of $D_{n}(4000)$ for some simple galaxy SFHs using stellar population synthesis (SPS) models at two different metallicities. While there are some variations with metallicity, in general $D_{n}(4000)$ increases for smaller ratios of current to past-averaged SFRs (i.e., "older" galaxies). One complication in the use of $D_{n}(4000)$ is that it is only measured within the spectroscopic aperture, and so galaxies with moderate bulge-to-disk ratios may have $D_{n}(4000)$ overestimated; this is clearly the case for a small number of galaxies in this sample (see also Kauffmann et al. 2007).

\section{2. $\operatorname{IR} X$}

A common and robust attenuation indicator is the ratio of IR dust emission to UV stellar emission [the so-called infrared excess, IRX $\left.=\log \left(L_{\text {dust }} / L_{\mathrm{UV}}\right)\right]$. It is independent of and a qualitatively different kind of measure than various optical measures based on a color excess (e.g., the Balmer decrement). This indicator is predicated on the idea that the UV emission measures the amount of transmitted flux from young stars, while the IR emission (here $\lambda \lambda 8-1000 \mu \mathrm{m}$ ) measures the amount of UV flux from young stars absorbed by dust and reradiated in the thermal IR (Buat 1992; Calzetti et al. 1994; Kennicutt 1998; Gordon et al. 2000). Under these simplistic assumptions the attenuation in the $\mathrm{UV}$ (in magnitudes) can be written $A_{\mathrm{UV}}=2.5 \log \left(10^{\mathrm{IRX}}+1\right)$. Light will be absorbed at wavelengths other than the UV, but the combination of dust attenuation curves that rise steeply toward 
the blue with blue intrinsic spectra has justified these assumptions when considering intensely star-forming galaxies. For many of the galaxies we consider this approximation may not be validspecifically, the fraction of the IR luminosity due to the heating of dust by UV light may vary between galaxies - but following Meurer et al. (1999) we define

$$
\hat{A}_{\mathrm{IRX}}=2.5 \log \left(\eta 10^{\mathrm{IRX}}+1\right),
$$

with $\eta=1 / 1.68$ for use when comparing the IRX we determine to other measures of attenuation in magnitudes. The parameter $\eta$ gives the fraction of the IR luminosity that comes from dustabsorbed UV light produced by young stars. Note that other conversions between IRX and attenuation are available (e.g., Bell 2002; Buat et al. 2005) but are generally consistent with this formulation, which has the advantage of being physically motivated and simply understood. The value of $\eta$ that we choose is similar to that chosen by Buat et al. (2007) and is consistent with Meurer et al. (1999), Bell (2003), Hirashita et al. (2003), and Iglesias-Páramo et al. (2006). In Johnson et al. (2007a) we attempt to estimate $\eta$ for each galaxy.

In detail, several modifications to the naive interpretation of IRX as a measure of attenuation are possible. First, there is the aforementioned effect of some fraction of the light being absorbed at wavelengths other than $\lambda \sim 1400 \AA$, which will vary depending on the SFH of the galaxy. Second, the possibility of different stellar populations within the galaxy being attenuated by different amounts of dust must be considered (Charlot \& Fall 2000). A third and related complication is the effect of the relative geometry of the stars and the dust, on both large and small scales (Witt \& Gordon 2000; Buat \& Xu 1996), although Gordon et al. (2000) find that for young stellar populations IRX is a good measure of the UV attenuation for a range of dust geometries and extinction laws, but they do not consider the effects of inclination in realistic disks on this measure (e.g., Pierini et al. 2004). Finally, older, blue horizontal-branch stars may contribute to the UV flux, and AGNs may contribute to both the UV and IR emission. However, despite these complications it appears that, at least for galaxies with $D_{n}(4000)<1.7$, IRX is an accurate dust estimator that increases monotonically with $A_{\mathrm{FUV}}$ for a given SFH (Johnson et al. 2007a).

It is important to clarify the meaning of IRX as used throughout this paper. For simplicity, and to conform with the convention in the literature, we use IRX defined by the total IR and UV luminosities [IRX $=\log \left(L_{\mathrm{dust}} / L_{\mathrm{UV}}\right)$, where $L_{\mathrm{dust}}$ and $L_{\mathrm{UV}}$ are defined in $\S 2.3]$. The issues mentioned above regarding the relation of IRX to the true FUV attenuation are considered separately in Johnson et al. (2007a).

\subsection{Color-Magnitude Diagram}

The CMD for galaxies is a fundamental observational tool in the study of galaxy evolution. Neglecting the effect of dust (and to a lesser extent metallicity), the color coarsely measures the SFH of a galaxy, while the absolute magnitude in one of the redder bands measures the total stellar mass (again subject to the effects of dust attenuation and to a lesser extent the SFH). The CMD thus relates the current SF to the past integrated SF of galaxies. Baldry et al. (2004) and Blanton et al. (2003) definitively identified the bimodality of galaxies in this diagram at low redshift using the SDSS, with many galaxies having red or blue colors and few galaxies at intermediate color. Kauffmann et al. (2003b) and Brinchmann et al. (2004) have constructed the SFH and stellar mass analog of the CMD for $\sim 500,000$ SDSS galaxies. Studies of the evolution of the distribution of galaxies in the CMD (Bell
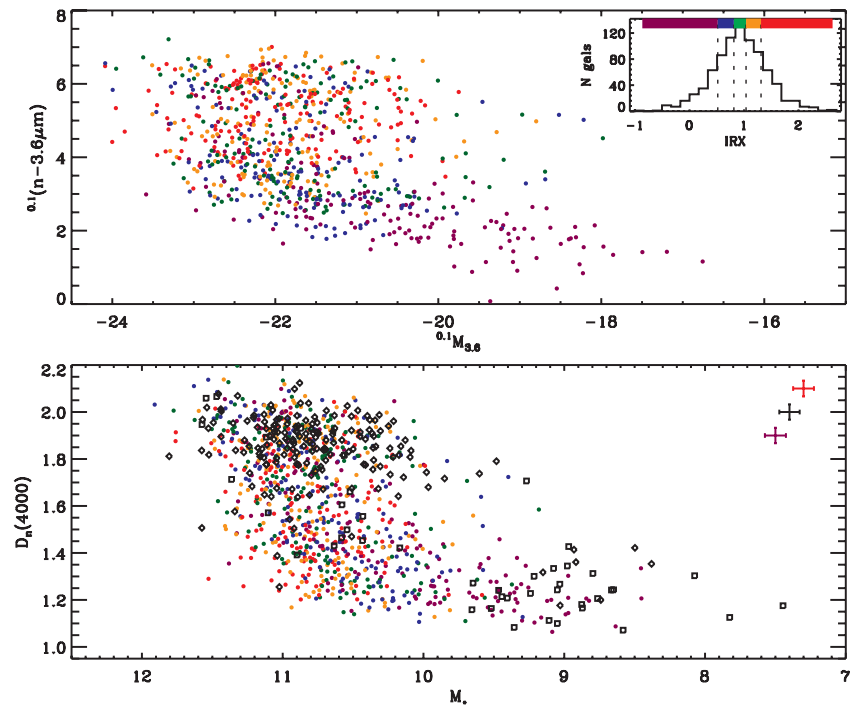

FIG. 2.-CMD for the sample detected at all wavelengths. Top: The ${ }^{0.1}(n-$ $3.6 \mu \mathrm{m})$ color vs. $M_{3.6}$. The color of each symbol encodes its IRX quintile, from lowest ( purple) to highest (red). The median photometric errors are smaller than the symbol size. The inset shows the distribution of IRX and the bins used to color-code the symbols. Bottom: Same as top, but with $D_{n}(4000)$ as the $y$-axis and $M_{*}$ from Kauffmann et al. (2003b) as the $x$-axis. Black diamonds show galaxies undetected in the UV or at $24 \mu \mathrm{m}$, while squares mark galaxies with $z<0.02$ or Petrosian radius greater than $11^{\prime \prime}$ in the $r$ band. Error bars show the median error for the lowest quintile ( purple) and highest quintile (red) of IRX, as well as the median error for the entire sample (black).

et al. 2004b; Faber et al. 2007) have identified an increase in the mass density of the red sequence from $z \sim 0.7$ to $z \sim 0.1$.

In Figure 2 we show the CMD of the sample galaxies. In the top panel we use the $n-3.6 \mu \mathrm{m}$ color; this is similar to the traditional CMDs using $u-r$ or $g-r$ (Baldry et al. 2004; Blanton et al. 2003), except that the use of the $n$ magnitude causes greater separation of the red and blue sequences (Wyder et al. 2007). The color of each symbol encodes its IRX quintile: red symbols are in the highest IRX quintile and tend to be located near the massive, high- $D_{n}(4000)$ edge of the blue sequence or on the red sequence, while purple symbols are in the lowest IRX quintile and are found primarily in the low-mass tail of the blue sequence (i.e., dwarfs). In the bottom panel we use $D_{n}(4000)$ as the measure of SFH. This measure is more directly related to SFH than the broadband color; as we show below the color is additionally affected by dust attenuation. The stellar mass estimates are from Kauffmann et al. (2003b) (§ 2.1). In this diagram we also show the location of galaxies undetected in the UV or at $24 \mu \mathrm{m}$; these are approximately $25 \%$ of the SDSS/MPA galaxies with $D_{n}(4000)>1.7$, as well as some dwarfs. Galaxies that do not pass the size cuts $[z>0.02$, $\left.R_{\mathrm{P}}(r)<11^{\prime \prime}\right]$ are also identified.

We clearly see the red and blue sequences, and their separation, in both diagrams. The blue sequence is tilted such that it becomes redder with increasing stellar mass. The degree of tilt, the scatter around the main sequences, and the evolution of both can be used to constrain the SFH of galaxies (Schweizer \& Seitzer 1992; Faber et al. 2007; Noeske et al. 2007; Schiminovich et al. 2007). There is also an increase in the average IRX with mass. Many of the galaxies in the "green valley" between the red and blue sequence appear to have large IRX. The accurate correction of observed colors for the presence of dust is thus a crucial step in determining the true tilt of the blue sequence for comparison to theories of galaxy formation. Attenuation is mass- (and SFR-) dependent, so inaccurate attenuation corrections can lead to incorrectly determined distributions of SFR with mass. 


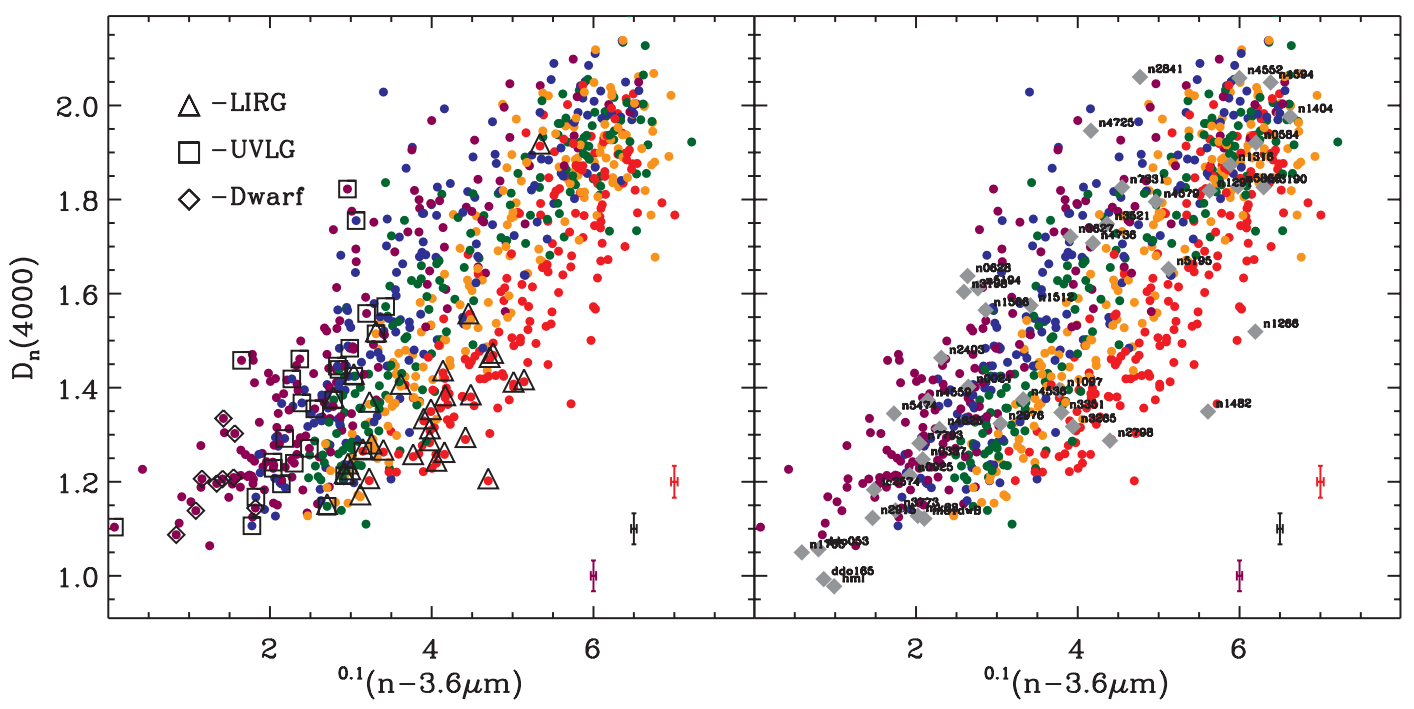

Fig. 3. - Left: Relation between IRX, $D_{n}(4000)$, and color with location of LIRGs, UVLGs, and dwarf galaxies marked (see $\S 2.5$ for definition of these classes). The color of each symbol indicates the IRX quintile, from lowest ( purple) to highest (red), as in Fig. 2. Error bars in the lower right show the median error for the lowest quintile ( purple) and highest quintile (red ) of IRX, as well as the median error for the entire sample (black). Right: Same as left, but with the location of galaxies from the SINGS and NGS surveys marked by gray diamonds.

\subsection{Dust-SFH-Color}

In Figure 3 we show the relation between IRX, $D_{n}(4000)$, and $n-3.6 \mu \mathrm{m}$ color for the sample of galaxies presented in this work. The color of each symbol indicates the IRX quintile. This diagram was introduced in Johnson et al. (2006). There is a clear relation between $D_{n}(4000)$ and color for the lowest quintiles of IRX (i.e., purple or blue symbols), although the relation becomes somewhat more scattered at high $D_{n}(4000)$. For larger values of IRX (i.e., orange or red symbols) the relationship between color and $D_{n}(4000)$ persists but is shifted to redder colors because of the reddening effect of the dust. Figure 3 shows how the color is additionally affected by the attenuation, as well as $\mathrm{SFH}$ - in this diagram the reddening vector is nearly horizontal — and how the conversion from color to $D_{n}(4000)$ in the CMD may be made. (i.e., by going from the top to the bottom panel in Fig. 2).

The locations of LIRGs, UVLGs, and dwarfs are shown in Figure $3 a$, while the locations of nearby galaxies drawn from the SINGS and GALEX NGS are shown in Figure $3 b$. The LIRGs clearly occupy the reddest region for a given $D_{n}(4000)$ and typically follow the sequence of galaxies with large IRX. The UVLGs are generally blue for their $D_{n}(4000)$ and have relatively low attenuation, although they do not follow the sequence of galaxies with the lowest IRX. The nearby galaxies cover the parameter space probed by the current sample reasonably well.

In Figure 4 we show SDSS gri composite images of galaxies in the sample, selected randomly from bins of $D_{n}(4000)$ and ${ }_{0.1}(n-3.6 \mu \mathrm{m})$ color. At high $D_{n}(4000)$ and relatively blue color we can see examples of galaxies with red bulges and blue starforming disks, highlighting the effect of aperture on the $D_{n}(4000)$ measurement (Kauffmann et al. 2007). At moderate $D_{n}(4000)$ and very red color there are many highly inclined, apparently red disks showing the effect of attenuation on color, while $D_{n}(4000)$ is relatively unaffected.

\section{IMPLICATIONS OF THE RELATION BETWEEN ATTENUATION, SFH, AND COLOR}

Having identified the relation between dust attenuation, SFH, and color as a useful diagnostic of galaxy properties, here we seek to explore the nature of the relation in more detail by considering different projections and a wider range of colors sampling the entire UV-optical SED. We also compare the relation to models that predict, separately, the effects of dust attenuation and $\mathrm{SFH}$ on color. In this way we can determine which UV-optical colors, in combination with $D_{n}(4000)$ and IRX, best constrain the models of attenuation and SFH. Finally, we parameterize the empirical relationship for these additional colors and examine the accuracy of the parameterization and the degree to which deviations are correlated with several additional physical properties of the galaxies.

\subsection{Extension to Additional Colors}

In Figure 5 we show $D_{n}(4000)$ versus color for a number of different broadband colors. The top panels show colors with a shortwavelength separation, while the middle panels show colors with a longer wavelength separation. As in Figure 3 the symbols are color-coded by their IRX quintile. We see a clear separation of the effects of dust and SFH on the colors with widely separated wavelengths. This behavior is discernible in the colors with a shorter wavelength separation as well (e.g., $g-r, u-g$ ), but the separation is not as clear. For each panel of Figure 5 the effect of dust attenuation is to move galaxies nearly horizontally along the color axis, since the effect of attenuation on $D_{n}(4000)$ is minimal (but see $\S 4.3$ ). In effect, the reddening vector is nearly horizontal. Thus, a relation between $D_{n}(4000)$ and color that appears for galaxies with low IRX ( purple symbols) will simply be translated along the color axis for galaxies with large IRX (red symbols) if the relation between $D_{n}(4000)$ and unattenuated color is nearly constant among galaxies. The size of the translation is constrained by IRX. The clear separation of $D_{n}(4000)$ and dust effects also implies a nearly invariant relation between $D_{n}(4000)$ and unattenuated color, at least for regions of the diagram with low scatter $\left[D_{n}(4000)<1.7\right]$.

There are some changes in the form of the dust-SFH color relation depending on the color used. In particular, at red $g-r$ color $(g-r \sim 0.9)$ there is saturation in the sense that $g-r$ color does not change significantly even as $D_{n}(4000)$ increases or for different values of IRX. This is in contrast to the $n-r$ and other long-wavelength baseline colors where there is a relatively large 


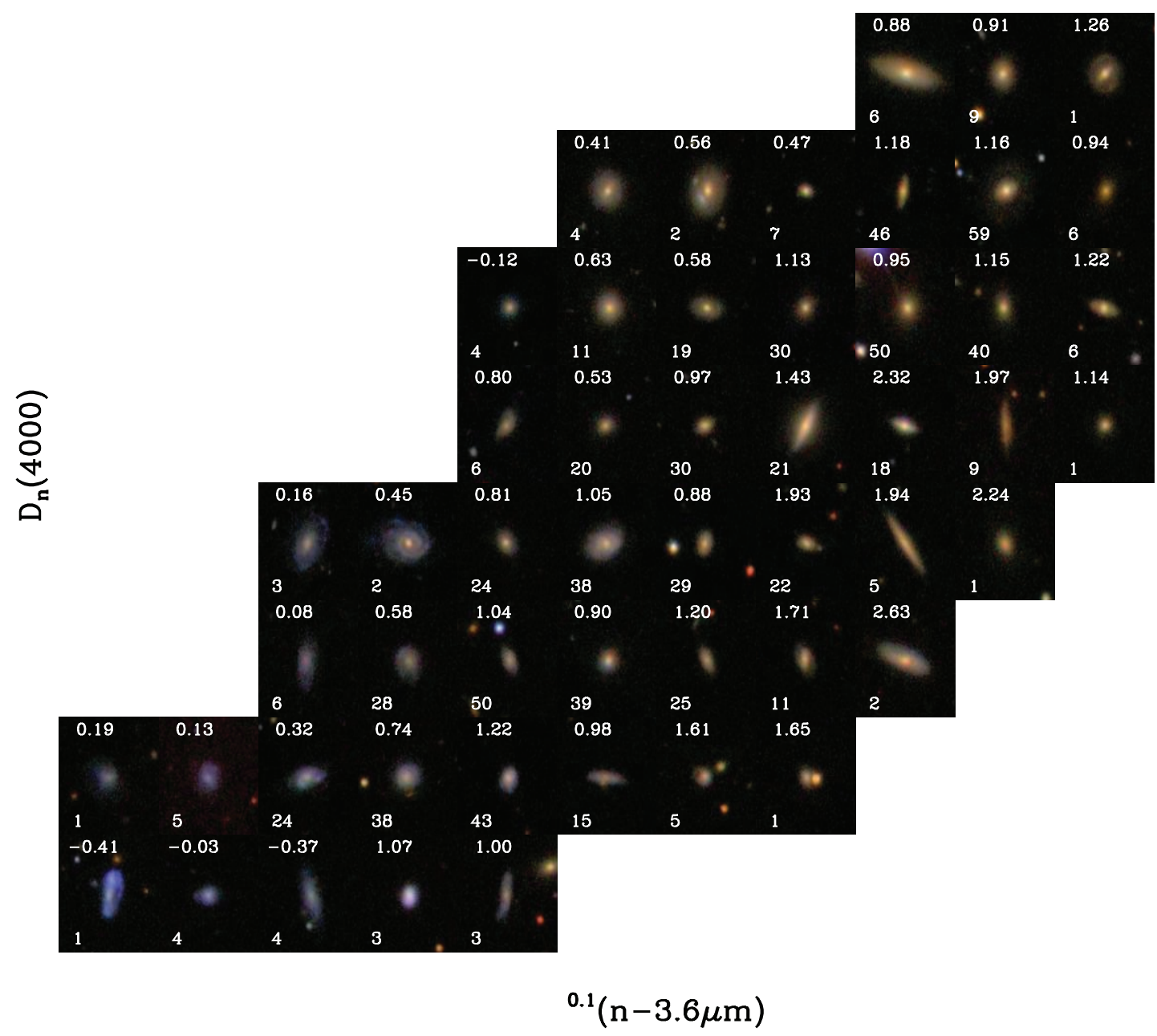

Fig. 4.-SDSS gri color-composite images of galaxies, organized in bins of $D_{n}(4000)$ and ${ }^{0.1}(n-3.6 \mu \mathrm{m})$ color. For each bin of $D_{n}(4000)$ or color (one-eighth and one-eleventh of the range, respectively) a galaxy is chosen at random for display. The top number in each image indicates the median IRX value for that bin, while the bottom number gives the number of galaxies in the bin.

variation in color for different values of $D_{n}(4000)$ and IRX. At low $D_{n}(4000)$ there is a difference in behavior of the $n-r$ and $n-3.6 \mu \mathrm{m}$ colors. The $n-3.6 \mu \mathrm{m}$ color shows a larger range at low $D_{n}(4000)$ than the $n-r$ color; this is because, for a given $D_{n}(4000)$ and large dust attenuations, the $r$-band flux is affected more than the $3.6 \mu \mathrm{m}$ flux, and so the $n-r$ color does not increase as rapidly as the $n-3.6 \mu \mathrm{m}$ color for increasing attenuations.

The trend of $D_{n}(4000)$ with $f-n$ color for a given narrow range of IRX is related to the effect of SFH on the UV spectral slope $\beta$ as described by Kong et al. (2004). Here we see that there is very little, if any, correlation of $f-n$ with $D_{n}(4000)$ for $D_{n}(4000)<$ 1.8 ; bands of nearly constant IRX appear vertical in the $D_{n}(4000)$ color plane, although there is a clear shift to redder colors for larger values of IRX. The IRX- $\beta$ relation is considered in more detail in Johnson et al. (2007b).

At $D_{n}(4000)>1.8$ the relation between $D_{n}(4000)$ and color is different; there is a ridge of galaxies for which $f-n$ decreases for increasing $D_{n}(4000)$. IRX appears to be significantly less correlated with $f-n$ for these galaxies than for those with $D_{n}(4000)<$ 1.8. As we see below ( $\S 4.1 .1)$ this ridge is close to the path followed by galaxies with a burstlike SFH and a small ratio of current to past-averaged SFR and traces the increasing importance of old blue stars in galaxies with very small ratios of young stars to old stars.

\subsubsection{SFH Models}

To aid in the interpretation of the relation between $D_{n}(4000)$ and SFH - and between SFH and color - we have constructed several SPS models of galaxy spectra using the Bruzual \& Charlot (2003) code. These models use the Chabrier (2003) IMF with the 1994 Padova tracks and have smooth, exponentially declining $\mathrm{SFR} \sim e^{-t / \tau_{\mathrm{SF}}}$ with no gas recycling. Colors (in the blueshifted filters) and $D_{n}(4000)$ are calculated directly from the resulting spectra. No dust attenuation has been applied. We show four of these models in Figure 5, having metallicity $Z / Z_{\odot}=0.4$ or 2.5 and $\tau_{\mathrm{SF}}=0.1$ or 1 Gyr. None of the models are starbursting in the sense that the ratio of the current to past-averaged SFR is always less than 1. A model with $\tau_{\mathrm{SF}}=10 \mathrm{Gyr}$ follows tracks similar to the $\tau_{\mathrm{SF}}=1$ Gyr models but never reaches $D_{n}(4000)>1.4$. In this diagram the models with $\tau_{\mathrm{SF}}=0.1$ are similar to a simple stellar population model. Models with different $\tau_{\mathrm{SF}}$ generally occupy the same region of $D_{n}(4000)$-color space at late times $(t>12$ Gyr), while models with different $Z$ have significantly different $D_{n}(4000)$ at late times. It is important to keep in mind that the $K$-corrections we have applied are derived from spectra based on the Bruzual \& Charlot (2003) models, so some care must be taken in drawing conclusions based on small effects in color. 

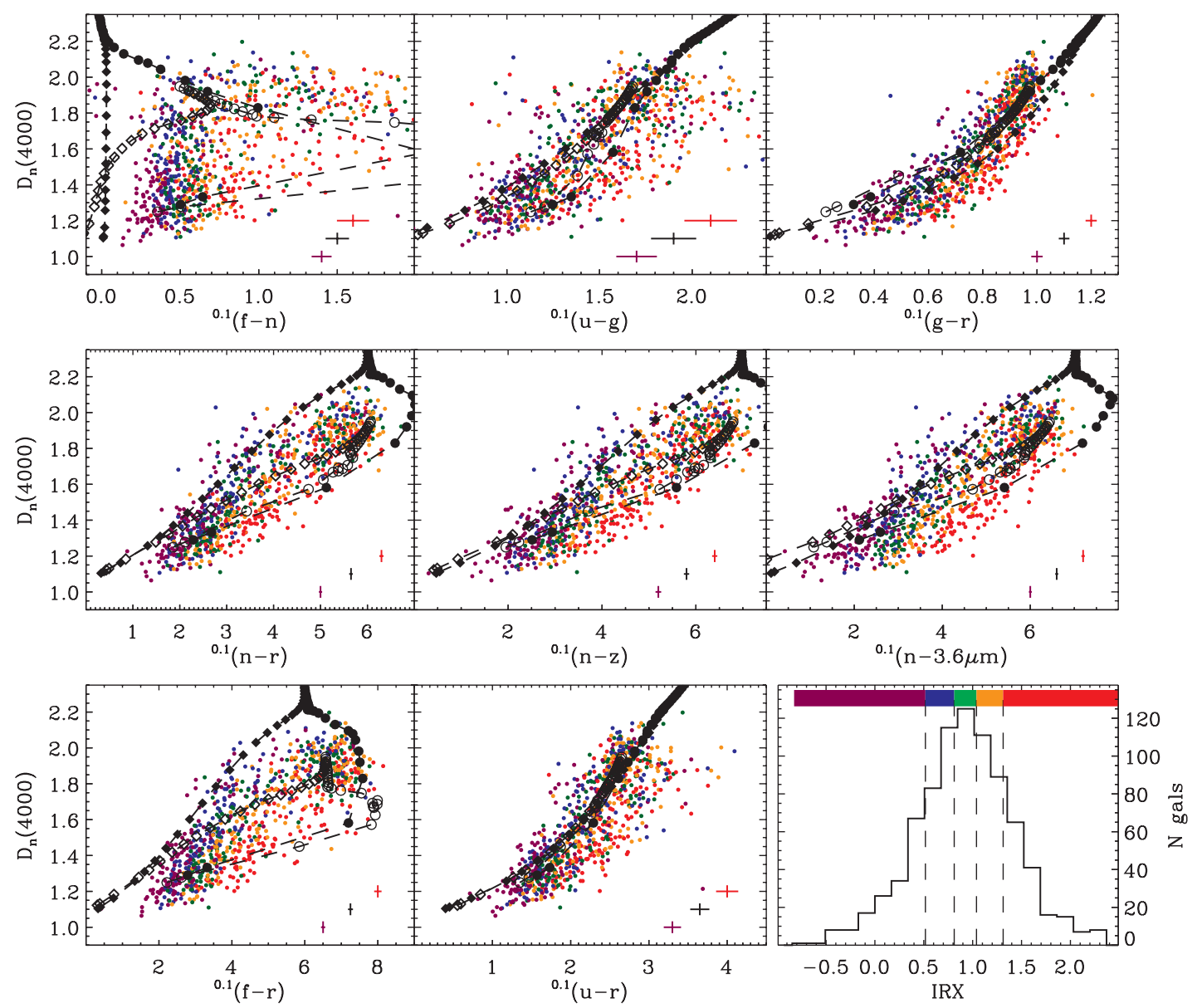

Fig. 5.- Relation between $D_{n}(4000)$ and color for different bins of IRX, for the colors $f-n, u-g, g-r, n-r, n-3.6, f-r$, and $u-r$. The bins of IRX used to color-code the symbols are shown in the bottom right panel. Error bars in the bottom right corner of each panel show the median error for the lowest quintile ( purple) and highest quintile ( red ) of IRX, as well as the median error for the entire sample (black). Overplotted in black are four Bruzual \& Charlot (2003) population synthesis models with $Z / Z_{\odot}=0.4$ (open symbols) and 2.5 ( filled symbols) and exponentially declining SFRs with time constants $\tau_{\mathrm{SF}}=0.1$ (circles) and $1 \mathrm{Gyr}$ (diamonds). The symbols are placed at intervals of $500 \mathrm{Myr}$, from 0.5 to $13 \mathrm{Gyr}$.

The zero-dust SPS models nevertheless yield several interesting insights. First, the models with different $\tau_{\mathrm{SF}}$ trace very different paths in the $D_{n}(4000)$ versus $f-n$ diagram. For the $\tau_{\mathrm{SF}}=1$ model the only change in $f-n$ occurs at late times for $Z / Z_{\odot}=$ 0.4 , even as $D_{n}(4000)$ steadily increases. This is consistent with the majority of galaxies at $D_{n}(4000)<1.7$ that, for a given small range in IRX, show little variation in $f-n$ color even as $D_{n}(4000)$ varies. The $\tau_{\mathrm{SF}}=0.1$ model exhibits strong variations in $f-n$ color as a function of time. The models start at ${ }^{0.1}(f-n) \sim 0.5$ and become very red, reaching a maximum of ${ }^{0.1}(f-n) \sim 2$ or $\sim 2.5$ for the high- and low-metallicity models, respectively, at $t \sim 1.5-2$ Gyr. After this time the models become bluer as the emission from evolved blue stars becomes dominant, while $D_{n}(4000)$ continues to increase. Although several SDSS/MPA galaxies fall along the $\tau_{\mathrm{SF}}=0.1$ track at low $D_{n}(4000)$ and redder colors, these galaxies also have significant IRX, such that they are incompatible with the zero-dust $\tau_{\mathrm{SF}}=0.1$ model. The attenuation-corrected ${ }^{0.1}(f-n)$ color of these galaxies would place them closer to the zero-dust $\tau_{\mathrm{SF}}=1$ model. In contrast, many of the galaxies with $D_{n}(4000)>1.8$ lie along a ridge that appears similar to the track of the fast-declining $\left(\tau_{\mathrm{SF}}=0.1\right)$ model, and these galaxies appear to have little relation between their color and IRX.

For the long-wavelength separation colors $\left[{ }^{0.1}(n-r),{ }^{0.1}(n-z)\right.$, and $\left.{ }^{0.1}(n-3.6 \mu \mathrm{m})\right]$ the zero-dust $\tau_{\mathrm{SF}}=0.1$ and 1 models fol- low much more similar tracks in $D_{n}(4000)$-color space. The galaxies in the lower IRX bins ( purple and blue symbols) follow a similar track, although there is more scatter for $D_{n}(4000)>1.7$. The galaxies with the largest IRX (orange and red symbols) are redder than the unattenuated models. Galaxies with significantly higher $D_{n}(4000)$ than any of the models for a given color are likely those for which the aperture effect causes an overestimate of global $D_{n}(4000)$ and have moderate bulge-to-disk ratios (e.g., Kauffmann et al. 2007). The increased scatter at $D_{n}(4000)>1.7$ may be due in part to increased scatter in the relation between IRX and attenuation for such galaxies (§3.2). In addition, and importantly, it is at $D_{n}(4000)>1.7$ where the models begin to diverge, and our simple relation between $D_{n}(4000)$, attenuation, and color may be broken because of scatter in the relation between $D_{n}(4000)$ and color. This scatter is in itself interesting, since it arises from different SFHs. It is only with robust, independent estimates of the attenuation that these small differences in the relation between color and $D_{n}(4000)$, corresponding to different forms of the $\mathrm{SFH}$, can be determined from observations. The variation between the models of the relation between $D_{n}(4000)$ and unattenuated color for these longer wavelength separation colors suggests that these colors (as opposed to the optical colors; see below) give some clue to the details of the SFH. This makes the plots involving these colors, and the $f-n$ color, the most useful for investigating the details of the SFH. 

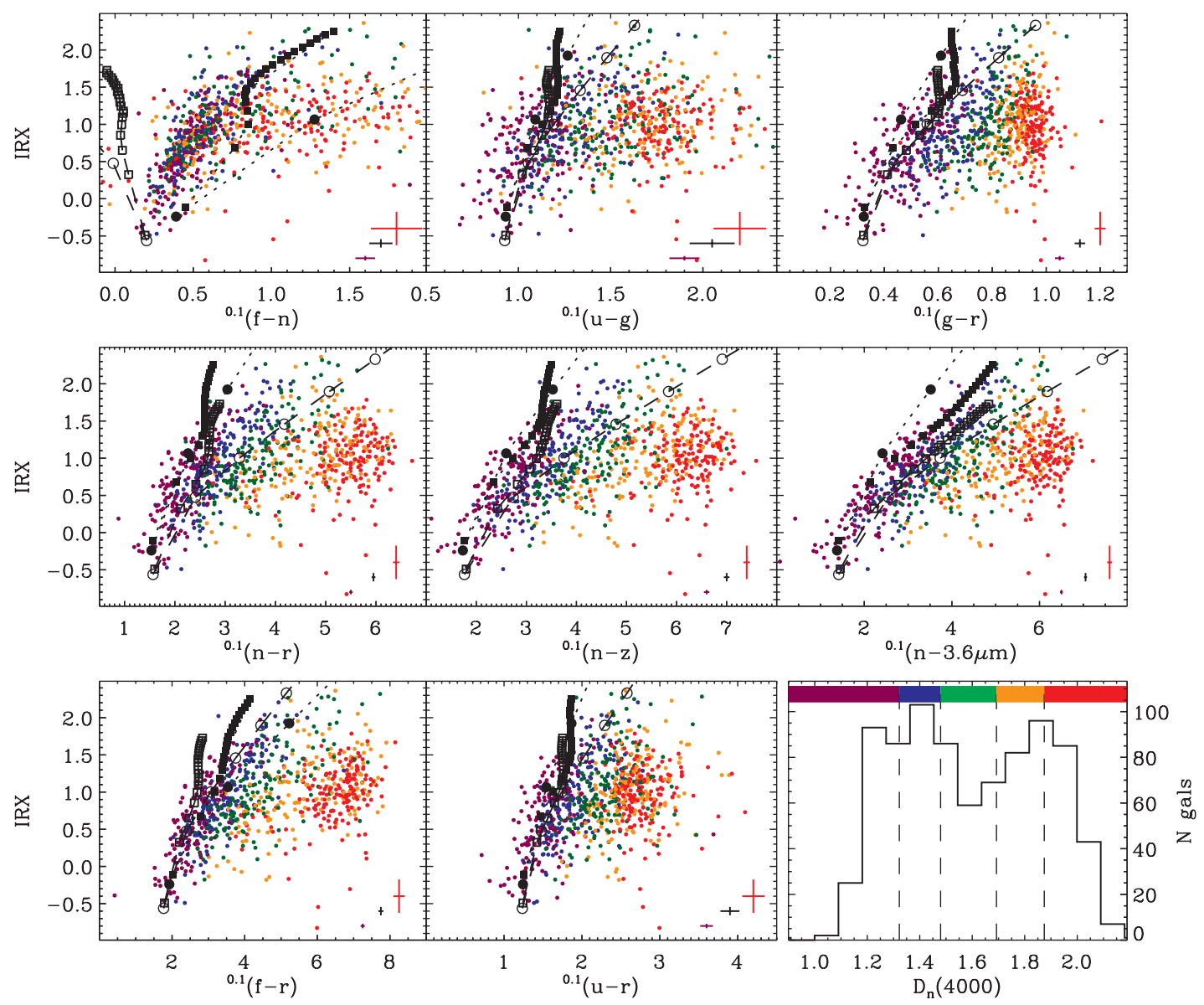

FIG. 6.- Relation between IRX and color for different bins of $D_{n}(4000)$ for the colors $f-n, u-g, g-r, n-r, n-z, n-3.6 \mu \mathrm{m}, f-r$, and $u-r$. The bins of $D_{n}(4000)$ used to color-code the symbols are shown in the bottom right panel. Error bars in the bottom right corner of each panel show the median error for the lowest quintile ( purple) and highest quintile (red) of $D_{n}(4000)$, as well as the median error for the entire sample (black). The black symbols and dashed lines show the effect of dust attenuation from Witt \& Gordon (2000) with a SHELL global geometry and for combinations of a MW (open symbols) or SMC ( filled symbols) extinction law and a clumpy (squares) or homogeneous (circles) local dust distribution. Symbols run from $\tau_{V}=0.1$ to 10 in steps of 0.5 . See text for details.

For the colors ${ }^{0.1}(u-g),{ }^{0.1}(g-r)$, and ${ }^{0.1}(u-r)$ the behavior of the models is different. These colors do not completely straddle the redshifted $4000 \AA$ break. For a given $D_{n}(4000)$ the different unattenuated models are nearly identical in these optical colors, even at $D_{n}(4000)>1.7$. Because the expected, unattenuated relation between $D_{n}(4000)$ and color appears nearly independent of the details of the SFH for these colors, deviations from this relation are more reliably interpreted as due to dust attenuation. Thus, dust attenuation would appear to be more easily inferred using the relation between $D_{n}(4000)$ and these colors than for the longer wavelength baseline colors that sample the UV [e.g., $\left.{ }^{0.1}(n-z)\right]$. However, these optical colors also have larger errors relative to the length of the reddening vector than the long-baseline colors, which makes the inference of attenuation more difficult.

\subsection{Binned by IRX}

In Figure 6 we show the relation between IRX and color for a number of different broadband colors. This is a different projection of the relation shown in Figures 3 and 5, and here the symbols are color-coded by $D_{n}(4000)$ quintiles.

For the $f-n$ color the relation between IRX and color is analogous to the IRX- $\beta$ relation (Meurer et al. 1999; Kong et al. 2004 ), where $\beta$ is the power-law slope of the UV spectrum. The correlation between IRX and $f-n$ has low scatter, and there is very little or no dependence on $D_{n}(4000)$ for $D_{n}(4000)<1.7$. Kong et al. (2004) have suggested that the additional effect of
SFH on UV color may account for the scatter in the IRX- $\beta$ relation. This would make the UV slope less reliable as a measure of attenuation in, e.g., high-redshift galaxies, without knowledge of the SFH. Gordon et al. (2000) argue that the effects of geometry may make the UV slope a poor measure of attenuation. We find that, for a variety of galaxies with $D_{n}(4000)<1.7$ observed in the local $z<0.3$ universe, the $f-n$ color is well correlated with IRX, although the slope is steep and therefore poorly constraining in the presence of significant errors in $f-n$ or $\beta$. However, for $D_{n}(4000)>1.7$ there is a large scatter in the relation, suggesting that the $f-n$ color for these galaxies is not primarily driven by the attenuation ( $\S 4.1 .1)$ or that IRX is not a good measure of the attenuation for these galaxies ( $\S 3.2$ ) (Johnson et al. 2007a). It is likely that both are true. The IRX- $\beta$ relation for this sample of galaxies is treated in more detail in Johnson et al. (2007b).

The $g-r$ and $u-g$ colors and near equivalents (such as $U-V$ ) are often used as indicators of red- and blue-sequence membership in the CMD (Bell et al. 2004b; Faber et al. 2007). We see here that they are somewhat affected by dust reddening (Bell et al. 2004a), and that care must be taken in defining the red and blue sequence, especially if there is evolution in the distribution of attenuation of galaxies as a function of redshift.

For the long-wavelength separation colors (e.g., $n-r$ or $n-3.6 \mu \mathrm{m}$ ) it is easy to see the effect of SFH on the color that was predicted by Kong et al. (2004) for the UV color. For a given low $D_{n}(4000)$ (i.e., much recent $\mathrm{SF}$, purple symbols) the relation 
between IRX and color is clear. This relation is closely related to the effective attenuation curve (e.g., Meurer et al. 1999). As $D_{n}(4000)$ increases (i.e., for different colored symbols in Fig. 6) the relation moves to redder color, but the slope and scatter do not change a great deal until the largest $D_{n}(4000)$. This shift to redder color at a given IRX is due to the redder intrinsic spectrum of a galaxy with larger $D_{n}(4000)$ (lower ratio of recent SFR to past-averaged SFR). However, at the highest $D_{n}(4000)$ the relation between IRX and color becomes more scattered.

\subsubsection{Dust Models}

To more explicitly show the relation of Figure 6 to dustattenuation laws, we have overplotted the relation between reddening and FUV attenuation given by the models of Witt \& Gordon (2000). These models include several different local and global dust geometries, as well as both Milky Way (MW) and Small Magellanic Cloud (SMC) extinction laws. The several global dust geometries consist of equally mixed dust and stars in a sphere (DUSTY), a shell of dust surrounding a sphere of stars (SHELL), and a cloud of dust at the center of the more extended stellar distribution (CLOUDY, where the radius of the dust is 0.69 times the radius of the stellar sphere). The local distribution is described as either homogenous ( $\mathrm{h}$ ) or clumpy (c). To place the model tracks in Figure 6 we convert the attenuation at $\sim 1400 \AA$ to IRX using equation (1). Gordon et al. (2000) show that this is a good approximation for all geometries with young stellar populations, although as mentioned in $\S 3.2$ for the older galaxies in the sample this approximation may not be valid. We calculate the color excess by taking the difference of the attenuations at the effective wavelength of the filters. The zero point in color is determined by averaging the color of the five bluest galaxies in the lowest $D_{n}(4000)$ quintile. However, for "older" galaxies that will have a redder intrinsic, unattenuated color the model curves can be translated along the color axis to match the zero dust color for that galaxy; in other words, the horizontal zero point of these tracks is somewhat arbitrary, depending on the unattenuated spectrum of the galaxy.

For clarity we only show the SHELL geometry in Figure 6. The CLOUDY model significantly underpredicts both IRX and color excess for $\tau_{V}<10$. While a few galaxies may be described by this model, the majority do not appear to be in this configuration, for the parameters used by Witt \& Gordon $\left(2000 ; r_{\text {dust }} / r_{\text {stars }}=\right.$ $0.69)$. The same is true of the DUSTY model with a clumpy local geometry. The MW dust-extinction law is in significant conflict with the observed IRX versus $f-n$ color relation. Because of the $2175 \AA$ bump in the MW extinction law (Stecher 1965; Draine \& Li 2007) the $f-n$ color becomes bluer with increasing $\tau_{V}$, a behavior not observed in the data. As can be seen in the top left panel of Figure 6, and as has been noted by other authors, the relation between UV slope and IRX is a very strong constraint on models of dust attenuation, particularly the extinction law. Note that some models that fit the data well for a given color do not fit the other colors. We find that the SMC extinction law with the SHELL geometry with clumpy or homogenous local geometries or the DUSTY model with homogenous local geometry provide the best matches to the trends of IRX with color excess in the data, although the DUSTY model gives significantly lower IRX and color excess for a given $\tau_{V}$ than the SHELL model.

\subsection{Binned by Color}

In Figure 7 we show the third projection of the dust-SFH-color relation, IRX versus $D_{n}(4000)$, for different ranges of $n-3.6 \mu \mathrm{m}$ color. This effectively shows the region of IRX and $D_{n}(4000)$ parameter space allowed for a given color. The extremely small amount of overlap of different color bins suggests that galaxies

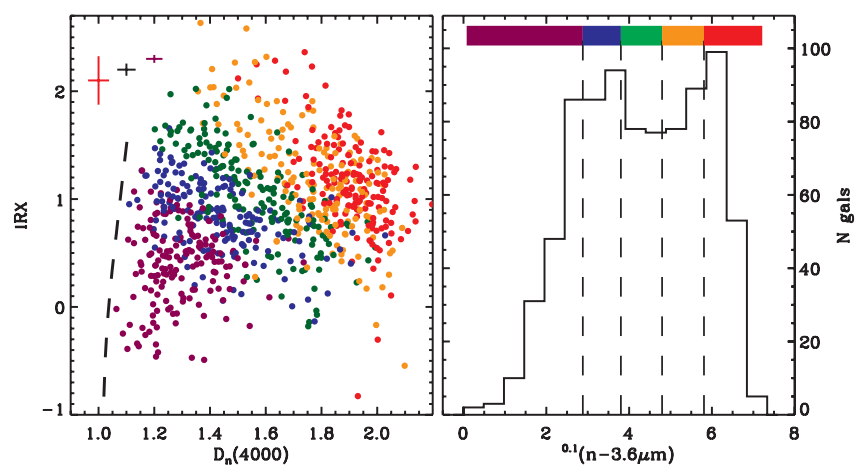

FIG. 7.-Left: Relation between IRX and $D_{n}(4000)$ for different bins of ${ }^{0.1}(n-$ $3.6 \mu \mathrm{m})$ color. The dashed line shows the effect of attenuation on $D_{n}(4000)$ for one of the models of MacArthur (2005). Error bars in the top left corner show the median error for the lowest quintile (purple) and highest quintile (red) of $0.1(n-3.6 \mu \mathrm{m})$, as well as the median error for the entire sample (black). Right: Histogram of ${ }^{0.1}(n-3.6 \mu \mathrm{m})$ color, showing the quintiles used to color-code the symbols.

follow nearly parallel "isocolor" lines in this diagram. Note that at high $D_{n}(4000)$ such isocolor lines do overlap, suggesting that an additional parameter may be necessary to explain the $n-3.6 \mu \mathrm{m}$ color of these galaxies. This diagram also shows the correlation (or lack thereof) between IRX and SFH in galaxies and is comparable to similar diagrams constructed for resolved galaxies in the local universe (Dale et al. 2007). However, one must again be cautious due to the effect of SFH on the interpretation of IRX as a measure of attenuation.

The envelope of lowest $D_{n}(4000)$ galaxies moves to slightly higher $D_{n}(4000)$ at higher IRX, so that there are no galaxies with significant dust attenuation and extremely low $D_{n}(4000)$. It is possible that galaxies undergoing recent $\mathrm{SF}$ in the local universe rarely have much dust. However, it is also quantitatively consistent with the increase in $D_{n}(4000)$ expected due to dust reddening, an indication that $D_{n}(4000)$ is not a perfectly "clean" indicator of SFH (MacArthur 2005). Using an approximation for effective optical depth, $\tau_{V}=\left[(5500 / 1390)^{-0.7} / \log e\right] \hat{A}_{\mathrm{IRX}}$, where we assume $\tau_{\lambda} \propto \lambda^{-0.7}$, we can compare a model of MacArthur (2005) to our sample in Figure 7. This is shown as a dashed line in Figure 7. This same trend can be seen in the data in Figure 5, where the envelope of low $D_{n}(4000)$ moves to larger values as the color (i.e., dust attenuation) increases. This shows the degree of reddening of $D_{n}(4000)$ for large values of attenuation and gives the deviation of the reddening vector from an exactly horizontal line in this diagram.

\subsection{Parameterization of the Relation}

The relation between attenuation, color, and SFH described above is determined by the dust-attenuation law and the correlation of $D_{n}(4000)$ with unreddened color. We leave a more rigorous derivation of a dust law to a separate work (Johnson et al. 2007b). Here we give a simple parameterization of the relation between IRX, color, and $D_{n}(4000)$ for a variety of colors. We fit using $\hat{A}_{\text {IRX }}$ as the measure of dust attenuation, since this is linear in color and should closely approximate the true $A_{\mathrm{FUV}}$ for relatively blue galaxies $(\S 3.2)$. The relation between $D_{n}(4000)$ and color is allowed to be linear or quadratic, based on visual inspection of Figure 5. We are here assuming that the relation between $D_{n}(4000)$ and the unreddened color is the same for all galaxies. Finally, after inspection of the fit residuals, we allow a cross-term whose origin is unclear but may be related to the change in the relation between $A_{\mathrm{FUV}}$ and $\hat{A}_{\mathrm{IRX}}$ as a function of SFH. Since the 
TABLE 2

Empirical Fits for Different Broadband Colors

\begin{tabular}{|c|c|c|c|c|c|c|c|c|c|c|c|c|c|}
\hline \multicolumn{7}{|c|}{ All Galaxies } & \multicolumn{7}{|c|}{$D_{n}(4000)<1.6$ AND $^{0.1}(n-r)<4(401$ GaLAXIES $)$} \\
\hline$A$ & $B$ & $C$ & $D$ & $E$ & $\sigma\left(\hat{A}_{\mathrm{IRX}}\right)$ & $\sigma(\operatorname{IRX})$ & $A$ & $B$ & $C$ & $D$ & $E$ & $\sigma\left(\hat{A}_{\mathrm{IRX}}\right)$ & $\sigma(\operatorname{IRX})$ \\
\hline \multicolumn{14}{|c|}{$y={ }^{0.1}(n-r)-2$} \\
\hline $1.61 \ldots \ldots \ldots \ldots \ldots . . . . .$. & -2.96 & 0.77 & $\ldots$ & $\ldots$ & 0.76 & 0.40 & 1.21 & -3.16 & 1.38 & $\cdots$ & $\ldots$ & 0.60 & 0.33 \\
\hline $1.48 \ldots \ldots \ldots \ldots \ldots \ldots$ & -1.12 & 0.76 & -2.50 & $\ldots$ & 0.74 & 0.38 & 1.24 & -2.12 & 1.36 & -4.65 & $\ldots$ & 0.57 & 0.32 \\
\hline $1.25 \ldots \ldots \ldots \ldots \ldots$ & -1.33 & 1.19 & $\ldots$ & -1.02 & 0.70 & 0.35 & 1.20 & -2.51 & 1.48 & & -1.05 & 0.59 & 0.32 \\
\hline $1.20 \ldots \ldots \ldots \ldots \ldots$ & -3.35 & 1.57 & 4.69 & -1.91 & 0.68 & 0.35 & 1.22 & -2.23 & 1.44 & -2.00 & -0.79 & 0.59 & 0.32 \\
\hline \multicolumn{14}{|c|}{$y==^{0.1}(n-z)-2$} \\
\hline $1.23 \ldots \ldots \ldots \ldots \ldots$ & -3.27 & 0.78 & $\ldots$ & $\ldots$ & 0.73 & 0.38 & 0.74 & -3.26 & 1.18 & $\ldots$ & $\ldots$ & 0.58 & 0.32 \\
\hline 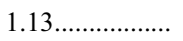 & -1.66 & 0.76 & -2.14 & $\ldots$ & 0.72 & 0.37 & 0.77 & -2.28 & 1.16 & -4.34 & $\ldots$ & 0.57 & 0.32 \\
\hline 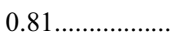 & -1.32 & 1.07 & $\ldots$ & -0.81 & 0.68 & 0.35 & 0.71 & -2.49 & 1.24 & $\ldots$ & -0.67 & 0.57 & 0.32 \\
\hline $0.65 \ldots \ldots \ldots \ldots \ldots$ & -2.79 & 1.36 & 4.22 & -1.53 & 0.66 & 0.35 & 0.75 & -2.20 & 1.20 & -2.90 & -0.35 & 0.57 & 0.32 \\
\hline \multicolumn{14}{|c|}{$y={ }^{0.1}(n-3.6 \mu \mathrm{m})-2$} \\
\hline $1.25 \ldots \ldots \ldots \ldots \ldots$ & -3.49 & 0.84 & $\ldots$ & $\ldots$ & 0.63 & 0.35 & 0.98 & -2.92 & 1.03 & & $\ldots$ & 0.46 & 0.28 \\
\hline $1.17 \ldots \ldots \ldots \ldots \ldots$ & -2.24 & 0.83 & -1.64 & $\ldots$ & 0.62 & 0.32 & 1.00 & -2.28 & 1.02 & -2.87 & $\ldots$ & 0.46 & 0.27 \\
\hline $0.98 \ldots \ldots \ldots \ldots \ldots \ldots$ & -1.89 & 1.02 & $\ldots$ & -0.63 & 0.59 & 0.31 & 0.98 & -2.65 & 1.05 & $\ldots$ & -0.25 & 0.46 & 0.27 \\
\hline $0.94 \ldots \ldots \ldots \ldots \ldots .$. & -2.80 & 1.16 & 2.51 & -1.02 & 0.58 & 0.31 & 1.00 & -2.27 & 1.02 & -2.81 & -0.02 & 0.46 & 0.27 \\
\hline \multicolumn{14}{|c|}{$y={ }^{0.1}(g-r)-2$} \\
\hline $10.23 \ldots \ldots \ldots \ldots \ldots$ & -3.17 & 5.75 & $\ldots$ & $\ldots$ & 0.74 & 0.38 & 10.60 & -3.71 & 5.99 & $\ldots$ & $\ldots$ & 0.67 & 0.36 \\
\hline $10.47 \ldots \ldots \ldots \ldots \ldots$ & -3.63 & 5.90 & 0.51 & $\ldots$ & 0.74 & 0.38 & 10.46 & -2.85 & 5.89 & -3.67 & $\ldots$ & 0.67 & 0.36 \\
\hline $10.35 \ldots \ldots \ldots \ldots \ldots$ & -4.34 & 5.85 & $\ldots$ & -1.05 & 0.74 & 0.38 & 10.84 & -6.64 & 6.15 & 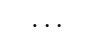 & -2.10 & 0.67 & 0.36 \\
\hline 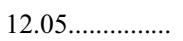 & -10.59 & 6.96 & 2.76 & -4.44 & 0.74 & 0.38 & 10.38 & -1.98 & 5.84 & -4.18 & 0.54 & 0.67 & 0.36 \\
\hline
\end{tabular}
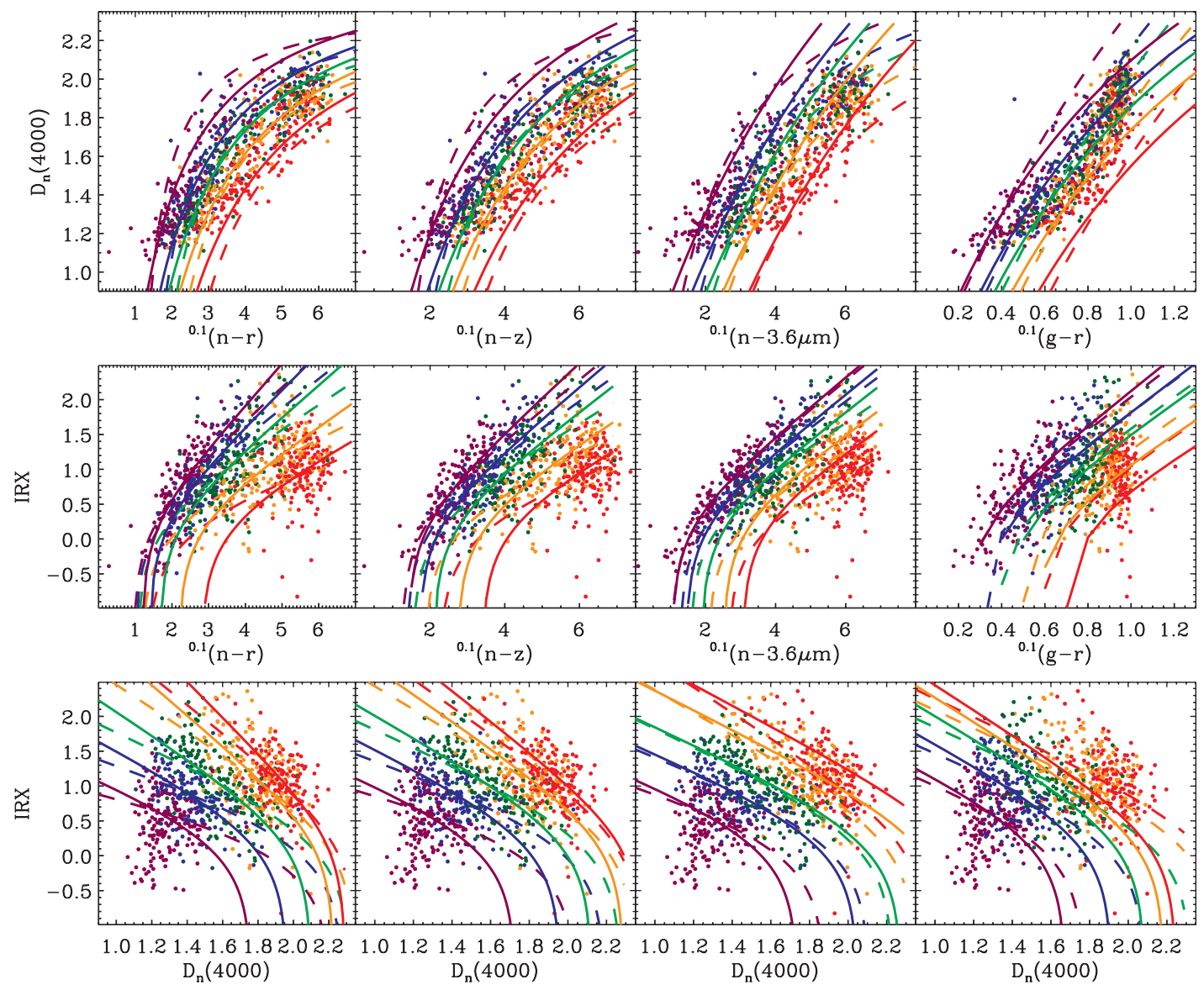

FIG. 8. - Fits of Table 2 overplotted on the three projections of the relation between IRX, $D_{n}(4000)$, and color. From left to right the colors used are $\left.{ }^{0.1}(n-r)\right)^{0.1}(n-z)$, ${ }^{0.1}(n-3.6 \mu \mathrm{m})$, and ${ }^{0.1}(g-r)$. The solid lines show the fits determined from galaxies with $D_{n}(4000)<1.6$ and ${ }^{0.1}(n-r)<4$, while the dashed lines show the fits determined from the entire sample. 
errors in $\hat{A}_{\text {IRX }}$ are larger than those in color or $D_{n}(4000)$, we treat $\hat{A}_{\text {IRX }}$ as the dependent variable. Thus, we fit

$$
\hat{A}_{\mathrm{IRX}}=A+B x+C y+D x^{2}+E x y,
$$

where $x=D_{n}(4000)-1.25$ and $y$ is the $\mathrm{AB}$ color minus 2 . The $A$ term thus provides the typical attenuation for galaxies in the sample with $D_{n}(4000)=1.25$ and a color of $\sim 2$. The results of these fits are given in Table 2 for several different colors. We have also conducted fits after restricting the sample to $D_{n}(4000)<1.6$ and ${ }^{0.1}(n-r)<4$. This allows us to ignore the effect of "older" galaxies for which $\hat{A}_{\mathrm{IRX}}$ is likely not a good indicator of attenuation and dust reddening may play a smaller role in determining the color than, e.g., metallicity, AGNs, or blue evolved stars. The inclusion of these high- $D_{n}(4000)$ galaxies may bias the fits, and when considering only blue-sequence galaxies we encourage the reader to use the fits restricted to $D_{n}(4000)<1.6$.

The fits including a cross-term $(E)$ but without a quadratic term in $D_{n}(4000)(D=0)$ are shown in Figure 8 along with the sample galaxies. This figure shows fits for both the entire sample and the $D_{n}(4000)<1.6$ subsample, in all three projections of the relation, and for the four colors given in Table 2. Because the displayed fits do not include $D$ term, the curvature seen in all colors is due to the cross-term, although for ${ }^{0.1}(n-3.6 \mu \mathrm{m})$ the fit to galaxies with $D_{n}(4000)<1.6$ appears very linear. The cross-term also causes the lines for different $\hat{A}_{\mathrm{IRX}}$ values to not be parallel. Despite the comparable dispersion in the residuals, the fits using other combinations of terms are not as well behaved. Residuals of the fits to the entire sample of galaxies are shown as a function of $D_{n}(4000)$ and color in Figure 9. It is possible that additional parameters play a role in the relation. For example, the metallicity of galaxies may have a significant additional effect on the color beyond the effects of SFH and attenuation. As a preliminary investigation of this possibility we show the residuals from the fit using ${ }^{0.1}(n-3.6 \mu \mathrm{m})$ plotted against other galaxy parameters in Figure 10. These parameters are the gas-phase metallicity as determined by Tremonti et al. (2004), the stellar mass from Kauffmann et al. (2003b), the SFR as determined by Brinchmann et al. (2004; where we use the median of the probability distribution function for the global SFR), and $\hat{A}_{\text {IRX. }}$. The strongest trend is with $\hat{A}_{\text {IRX }}$, the very quantity being fit. This is a result of the dispersion in the relation being a large fraction of the range and precludes detailed analysis of the residuals.

\subsection{Applications and Implications for Future Observations and Models}

The $\hat{A}_{\text {IRX }}$-color- $D_{n}(4000)$ relation may be used to determine the attenuation given $D_{n}(4000)$ and a color. Such derived attenuations may then be used to correct SFR indicators at other wavelengths. While $D_{n}(4000)$ is already a coarse measure of the SF activity of galaxies, it is sensitive to older stars than the $f$ band or $\mathrm{H} \alpha$ luminosity. The accuracy of the attenuation correction derived from the fits and applied to $\mathrm{UV}$ or $\mathrm{H} \alpha$ luminosities then depends on whether the most recently formed stars are affected by the same dust as those stars contributing to the long-wavelength separation color [as well as the accuracy of the fit for the chosen color and whether the form of the relation between $D_{n}(4000)$ and color varies with changes in the details of the SFH]. Treyer et al. (2007) have compared the UV fluxes corrected for attenuation using these fits to the $\mathrm{H} \alpha$-derived SFR. They find good agreement, although there is a trend of the differences with the SFR. A direct comparison of the UV+IR-derived SFR to the emission-linederived SFR is given in a separate work (Johnson et al. 2007a).
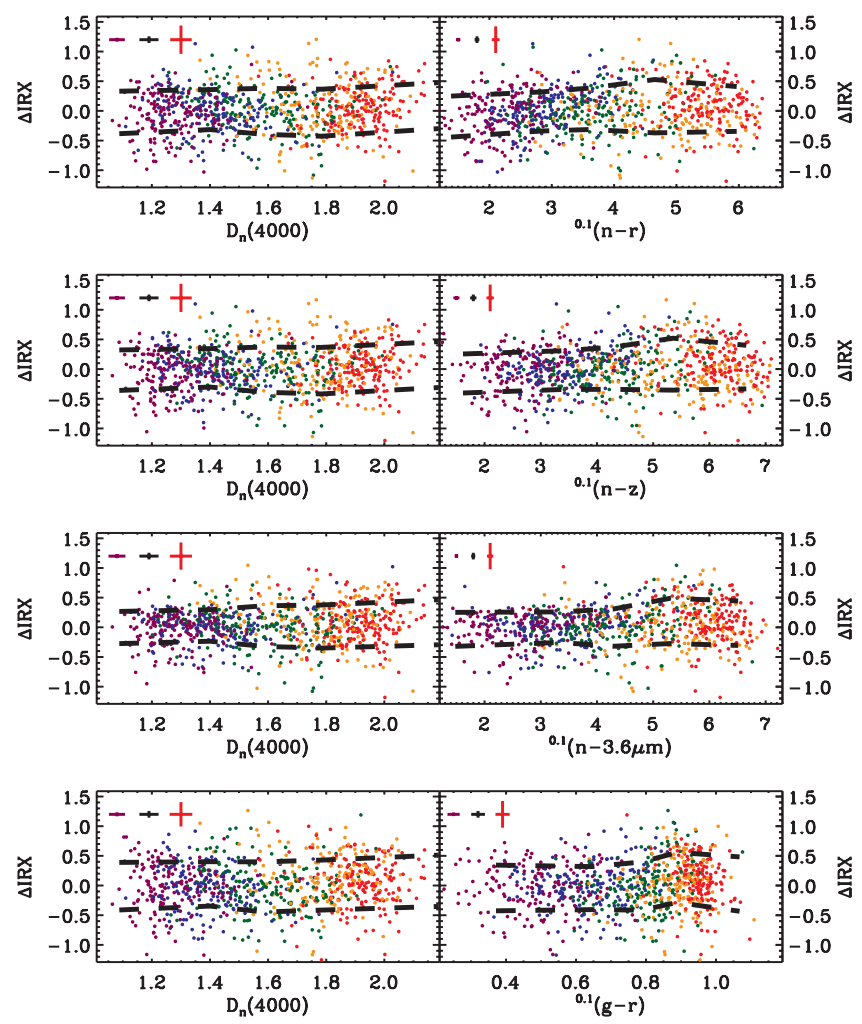

FIG. 9.- Residuals from the fit ( Table 2) for each color, plotted vs. $D_{n}(4000)$ and color. The residuals shown are from the fit to the entire sample including the $A, B, C$, and $E$ terms in Table 2. Dashed lines show the standard deviation of the residuals. The colors of the symbols in the left panels give the quintile of the observed color, while the colors of the symbols in the right panels give the $D_{n}(4000)$ quintile (see Fig. 6, bottom right panel).

Here we compare the accuracy of this method for attenuation correction to methods based on emission lines or the UV color. The standard deviations of the residuals from the fits, in terms of both $\hat{A}_{\text {IRX }}$ and IRX, are given in Table 2 . For the ${ }^{0.1}(n-3.6 \mu \mathrm{m})$ color these are 0.5 in $\hat{A}_{\mathrm{IRX}}$ and 0.3 in IRX. Attenuation may also be estimated from the Balmer decrement $A_{\text {Balmer }}=2.5 \log \left[\left(L_{\mathrm{H} \alpha} / L_{\mathrm{H} \beta}\right) / 2.88\right]$, where $L_{\mathrm{H} \alpha}$ and $L_{\mathrm{H} \beta}$ are the $\mathrm{H} \alpha$ and $\mathrm{H} \beta$ line luminosities, respectively (Kennicutt 1998; Johnson et al. 2007a). The ratio $\hat{A}_{\mathrm{IRX}} / A_{\text {Balmer }}$ is $\sim 3-5$ (Johnson et al. 2007a). Thus, to obtain a similar error in $\hat{A}_{\mathrm{IRX}}$ from the Balmer decrement requires $\mathrm{S} / \mathrm{N} \gtrsim 10$ in both the $\mathrm{H} \alpha$ and $\mathrm{H} \beta$ emission lines (or an equivalent combination of $\mathrm{S} / \mathrm{N}$ in the two lines). Another common method of attenuation correction is provided by the $A_{\mathrm{FUV}}-\beta$ relation (e.g., Meurer et al. 1999), where $\beta$ is the UV spectral slope (similar to the $f-n$ color). Seibert et al. (2005) quote a scatter of $\sigma\left(A_{\mathrm{FuV}}\right)=0.89$ in this relation when a range of galaxy types is considered. One must also consider the large slope in the relation between $\beta$ and $A_{\mathrm{FuV}}$. An uncertainty of 0.1 in $f-n$ color (due to photometric errors or uncertainty in the $K$-correction) leads to an uncertainty of $\sim 0.8$ in $A_{\mathrm{FuV}}$, even in the absence of scatter in the $A_{\mathrm{FuV}}-\beta$ relation. Such uncertainties in color [and $D_{n}(4000)$ ] are much less important for the $\hat{A}_{\mathrm{IRX}}$-color$D_{n}(4000)$ relation, especially when UV-optical colors are used that have large-wavelength separation and relatively small errors.

Besides correcting SFR measures at various wavelengths for dust attenuation, it may be interesting to consider the distribution of dust attenuations themselves - derived from the $\hat{A}_{\text {IRX }}$-color$D_{n}(4000)$ relation - as a probe of the SF in galaxies. The attenuation may be expected to trace the product of metallicity and gas surface density (Bell 2003; Johnson et al. 2007b), which are 


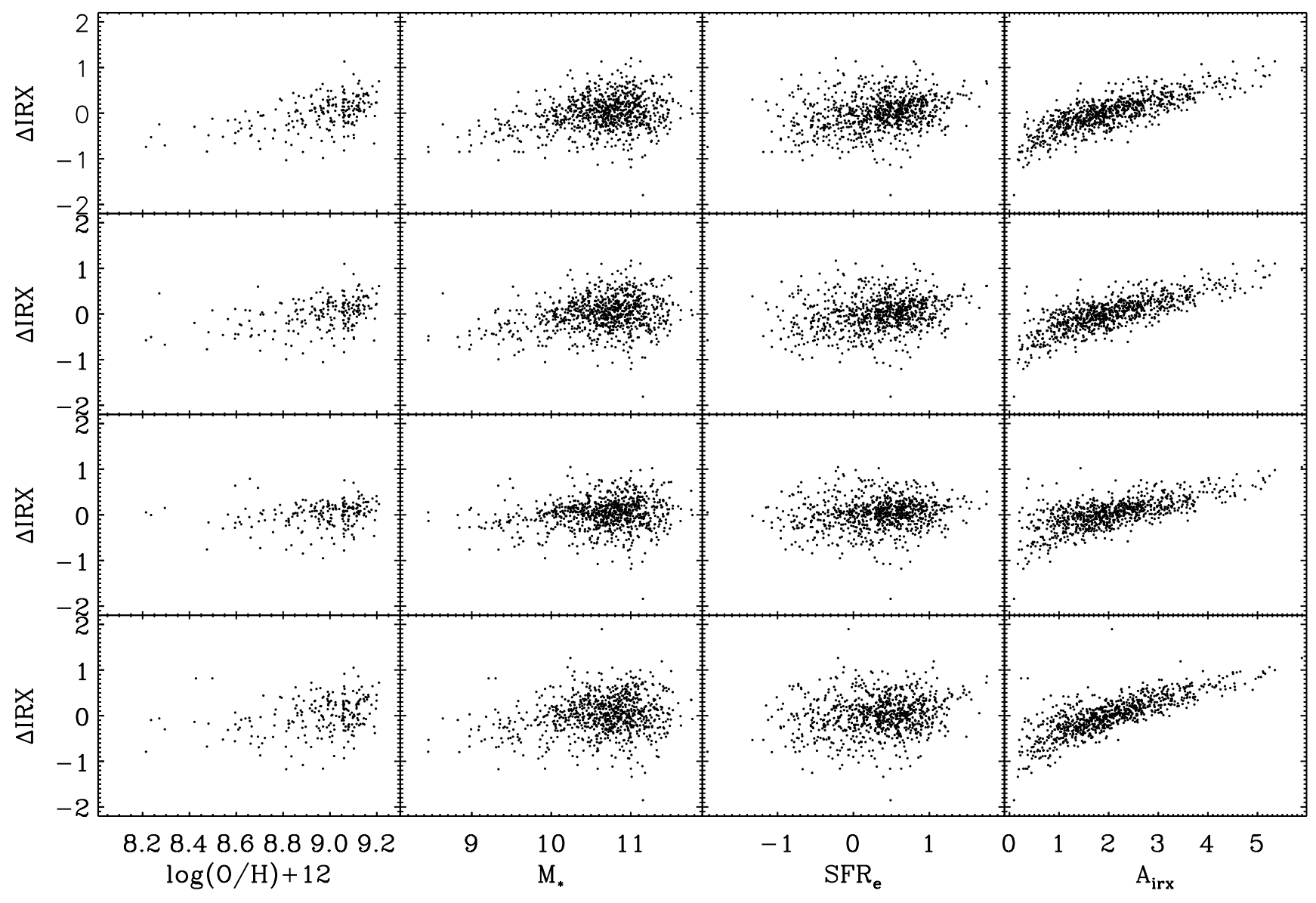

FIg. 10.- Residuals from the fit to the full sample, including the $A, B, C$, and $E$ terms in Table 2, plotted against other galaxy parameters. The colors used in the fit are (top to bottom $)^{0.1}(n-r),{ }^{0.1}(n-z),{ }^{0.1}(n-3.6 \mu \mathrm{m})$, and ${ }^{0.1}(g-r)$. The residuals are plotted against (left to right) the gas-phase metallicity (where available), the stellar mass, the optically determined SFR (from Brinchmann et al. 2004), and $\hat{A}_{\text {IRXX }}$.

both key diagnostics of the evolution of galaxies (e.g., Martin et al. 2007). The fit presented in $\S 4.4$ can be used to estimate the dust attenuation for large samples of galaxies, at a range of redshifts; where $D_{n}(4000)$ and a broadband color are known high $\mathrm{S} / \mathrm{N}$ emission lines are not necessary.

Several future observatories will add to the IR view of the universe provided by Spitzer, including AKARI (formerly Astro-F; Murakami 1998) and the proposed Wide-Field Infrared Survey Explorer (WISE; Mainzer et al. 2005). In particular, the flux limit of WISE at $23 \mu \mathrm{m}(\sim 2.6 \mathrm{mJy}, 5 \sigma)$ will enable the detection of $\sim 20 \%$ of SDSS/MPA galaxies at this wavelength (i.e., $10^{5}$ galaxies), although these will be primarily dusty star-forming galaxies. The parameterization of the relation given above will provide a context for this much larger sample of galaxies that will be strongly affected by selection effects in the IR. Combined with GALEX imaging over the SDSS footprint, the large number of galaxies can be used to investigate deviations from the relation as a function of, e.g., AGN activity or morphology; understanding such deviations will lead to improved modeling of attenuation and knowledge of the SFH. Such a large number of galaxies will also provide an unbiased view of the properties of rare types of galaxies (e.g., ULIRGs), which may or may not follow the $\hat{A}_{\text {IRX- }}$ color- $D_{n}(4000)$ relation derived here for more common galaxies. LIRGs at $z=0.5$ will be detectable by WISE over the entire sky at $23 \mu \mathrm{m}$, while the $12 \mu \mathrm{m}$ band of WISE will be able to probe the rest-frame $8 \mu \mathrm{m}$ PAH emission of these galaxies. Furthermore, nearly all SDSS/MPA galaxies will be detected in the shortest wavelength band $(\sim 3.3 \mu \mathrm{m})$. Such NIR photometry, when com- bined with rest-frame near-UV photometry, leads to the clearest relation between broadband color, $D_{n}(4000)$, and IRX, making accurate determinations of IRX possible for $\sim 10^{5}$ galaxies.

Finally, this parameterized relation between $D_{n}(4000)$, IRX, and color will have to be reproduced by the next generation of galaxy models that seek to self-consistently treat dust absorption and emission in the context of SPS modeling (Jonsson 2006; Li et al. 2007; Silva et al. 1998). The empirical relation given above provides an important diagnostic for such models, since it links in a relatively straightforward way the stellar populations, the absorption of the light of these populations by dust, and the subsequent reemission of that light by dust at IR wavelengths.

\section{COLOR-COLOR RELATIONS AT HIGH REDSHIFT}

At high redshift $(z \sim 2)$ several authors have attempted to distinguish between the effects of attenuation and SFH on the colors of galaxies using Spitzer IRAC imaging of the rest-frame NIR (Wuyts et al. 2007; Kriek et al. 2006). These use a methodology similar to that of Kauffmann et al. (2003b) by comparing the locations of galaxies in color-color space to a locus of SPS models and attributing differences to dust reddening. Wuyts et al. (2007) have used the relatively reddening-insensitive photometric break strength at $4000 \AA$ [sampling both $D_{n}(4000)$ and the Balmer break] explicitly as one of the "colors," with the benefit that the direction of the reddening vector is then relatively well known. The determination of IRX, as in the present work, gives empirical constraints on the length of the reddening vector, lending support to this method for SFH determination. 


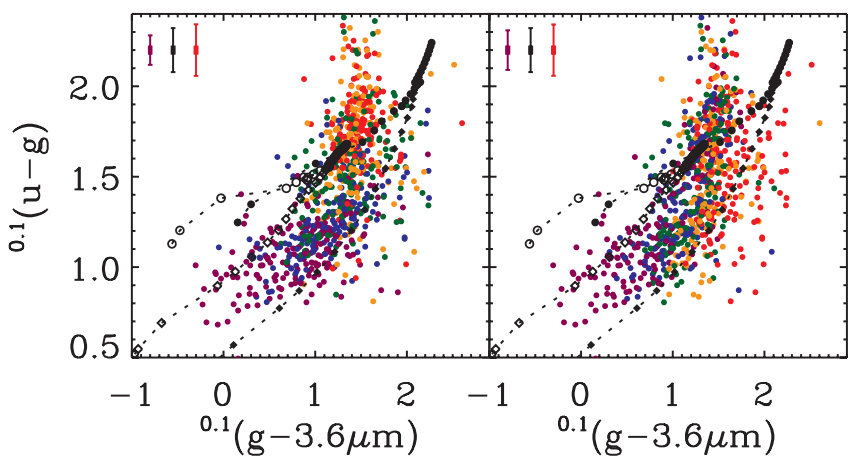

FIg. 11.-Color-color diagrams as available at high redshift using IRAC (Wuyts et al. 2007). On the left the symbols are color-coded by the $D_{n}(4000)$ quintile; on the right they are color-coded by IRX quintile. See Figs. 5 and 6 for the bins used for color-coding, respectively. The model symbols (black) are the same as in Fig. 5. Colored error bars at the top left corner of each panel show the median errors for the corresponding parameter bin, while the black error bars show the median for the entire sample.

In Figure 11 we show a color-color diagram for the sample galaxies that is quite similar to that constructed by Wuyts et al. (2007) for a number of galaxies at $z=1-3$, both in the colors plotted and in the locations of galaxies in the diagram. Galaxies tend to move toward redder colors in ${ }^{0.1}(g-3.6 \mu \mathrm{m})$ as IRX increases and are generally at high $D_{n}(4000)$ as ${ }^{0.1}(u-g)$ increases. As we have shown previously $(\S 4.1 .1)$, there is also some reddening of the $u-g$ color with IRX. For a large portion of the color space [i.e., ${ }^{0.1}(u-g)<1.5$ ], it is not possible to use the colorcolor diagram alone to distinguish between different model tracks and the amount of dust attenuation. With independent constraints on dust attenuation, and assuming that the population synthesis modeling yields the correct colors, it is possible to rule out highmetallicity $\left(Z / Z_{\odot}=2.5\right)$ exponentially declining models for a large proportion of the sample, although low-metallicity exponentially declining models are consistent with the data.

\section{CONCLUSIONS}

1. We have presented a low-redshift $(\bar{z}=0.1)$ context for the IR, UV, and optically derived dust attenuation, SF, and stellar mass properties of $\sim 1000$ homogeneously observed galaxies. The relation between SFH, attenuation, and color is a useful diagnostic of galaxy properties. We identified where special classes of galaxies lie in the color-color diagram of $D_{n}(4000)$ versus broadband color. We find that LIRGs occupy the same region of $D_{n}(4000)$, IRX, and color parameter space as dusty star-forming galaxies, of which many are likely to be highly inclined. We find that UVLGs occupy the same parameter space as a larger population of relatively attenuation-free, star-forming galaxies. However, UVLGs are not the least dusty galaxies for a given $D_{n}(4000)$. The distribution of galaxies in this parameter space, corrected for selection effects, can be compared to high-redshift samples and semianalytic models to determine the evolution of dust attenuation and to ensure that attenuation is being treated correctly in the models.

2. This relation between $D_{n}(4000)$, IRX, and color suggests that the colors of galaxies can be explained primarily as the contributions from the SFH (we have here assumed exponentially declining models) and the dust attenuation (Johnson et al. 2006). A comparison with stellar population synthesis and dust-attenuation models confirms that variation in $D_{n}(4000)$ and IRX causes changes in galaxy color quantitatively similar to those seen in the data. Detailed comparison of the exact form of the relation of $D_{n}(4000)$ and IRX to the color of galaxies has the power to con- strain these models. The UV color is especially sensitive to the form of the extinction law; a Milky Way extinction law (Cardelli et al. 1989) is not consistent with the change in UV colors of galaxies as a function of IRX for a range of relative star/dust geometries (but see Panuzzo et al. [2007] for a possible resolution involving the variation of attenuation with stellar age in galaxies).

For some colors [e.g., ${ }^{0.1}(u-r)$ ] the relation between $D_{n}(4000)$ and color appears to be independent of the specific SFH model assumed. Deviations of the observed galaxy colors from this relation may then be explained as reddening due to dust attenuation. However, for other UV-optical-NIR colors [e.g., ${ }^{0.1}(n-3.6 \mu \mathrm{m})$ ] the relation between $D_{n}(4000)$ and color depends on the specific form of the SFH, especially at moderate $D_{n}(4000)$ and color. In this case, independent constraints on the degree of reddening provided by IRX will allow different SFHs to be distinguished. Similarly, different SFH models follow extremely different tracks in the $D_{n}(4000)$ versus UV color plane, emphasizing the power of the UV color as a diagnostic of stellar populations.

3. We find through comparison with zero-dust stellar population synthesis models that a burstlike SFH leads to a relation between $D_{n}(4000)$ and UV color at late times that is similar to that seen for our sample galaxies at $D_{n}(4000)>1.7$. The colors of these galaxies do not appear to be highly correlated with IRX, suggesting that dust attenuation is not the dominant driver of UV color for these galaxies. This effect is likely caused by evolved blue stars. There is, however, a significant offset of the locus of sample galaxies from the Bruzual \& Charlot (2003) models of old stellar populations.

4. We have parameterized the relation between $D_{n}(4000)$, color, and IRX for a variety of colors. This allows the determination of one of these quantities provided the other two are known. Assuming a universal relation between $D_{n}(4000)$ and unattenuated color (as provided by most exponentially declining SF models), this parameterization may be used to infer the distribution of IRX in galaxies and the evolution thereof from high to low redshift. It may also be used to determine the dust attenuations for application to tracers of more recent SF. The best accuracy in inferred attenuations is achieved with the ${ }^{0.1}(n-3.6 \mu \mathrm{m})$ color; $A K A R I$ and the proposed WISE observatory, combined with $G A L E X$, will provide large samples of galaxies for which this color is available. This relation links dust absorption and emission and the stellar populations of galaxies. Models that seek to explain the optical and IR emission of galaxies self-consistently will need to reproduce this relation for low-redshift galaxies.

The anonymous referee provided extremely useful comments that resulted in substantial improvements to the paper. B. D. J. thanks S. Salim, A. Boselli, S. Boissier, and L. Cortese for helpful comments. B. D. J. was supported by NASA GSRP grant NNG05GO43H.

GALEX (Galaxy Evolution Explorer) is a NASA Small Explorer, launched in 2003 April. We gratefully acknowledge NASA's support for construction, operation, and science analysis for the GALEX mission, developed in cooperation with the Centre National d'Etudes Spatiales of France and the Korean Ministry of Science and Technology.

This work is based in part on observations made with the Spitzer Space Telescope, which is operated by the Jet Propulsion Laboratory, California Institute of Technology, under a contract with NASA. In particular, the publicly available Spitzer data obtained by the SWIRE team have been essential to this work. 
Funding for the SDSS and SDSS-II has been provided by the Alfred P. Sloan Foundation, the Participating Institutions, the National Science Foundation, the US Department of Energy, the National Aeronautics and Space Administration, the Japanese Monbukagakusho, the Max Planck Society, and the Higher Education Funding Council for England. The SDSS Web site is http://www.sdss.org. The SDSS is managed by the Astrophysical Research Consortium for the Participating Institutions. The Participating Institutions are the American Museum of Natural History, the Astrophysical Institute Potsdam, the University of Basel, the University of Cambridge, Case Western Reserve Uni- versity, the University of Chicago, Drexel University, Fermilab, the Institute for Advanced Study, the Japan Participation Group, Johns Hopkins University, the Joint Institute for Nuclear Astrophysics, the Kavli Institute for Particle Astrophysics and Cosmology, the Korean Scientist Group, the Chinese Academy of Sciences (LAMOST), Los Alamos National Laboratory, the Max Planck Institute for Astronomy (MPIA), the Max Planck Institute for Astrophysics (MPA), New Mexico State University, Ohio State University, the University of Pittsburgh, the University of Portsmouth, Princeton University, the United States Naval Observatory, and the University of Washington.

Abazajian, K., et al. 2004, AJ, 128, 502

\section{REFERENCES}

Baldry, I. K., Glazebrook, K., Brinkmann, J., Ivezić, Ž., Lupton, R. H., Nichol, R. C., \& Szalay, A. S. 2004, ApJ, 600, 681

Balogh, M. L., Morris, S. L., Yee, H. K. C., Carlberg, R. G., \& Ellingson, E. 1999, ApJ, 527, 54

Bell, E. F. 2002, ApJ, 577, 150 2003, ApJ, 586, 794

Bell, E. F., \& de Jong, R. S. 2001, ApJ, 550, 212

Bell, E. F., et al. 2004a, ApJ, 600, L11 2004b, ApJ, 608, 752

Bertin, E., \& Arnouts, S. 1996, A\&AS, 117, 393

Blanton, M. R., \& Roweis, S. 2007, AJ, 133, 734

Blanton, M. R., et al. 2003, ApJ, 594, 186

Brinchmann, J., Charlot, S., White, S. D. M., Tremonti, C., Kauffmann, G.,

Heckman, T., \& Brinkmann, J. 2004, MNRAS, 351, 1151

Bruzual, G., \& Charlot, S. 2003, MNRAS, 344, 1000

Buat, V. 1992, A\&A, 264, 444

Buat, V., \& Xu, C. 1996, A\&A, 306, 61

Buat, V., et al. 2005, ApJ, 619, L51 2007, ApJS, 173, 404

Calzetti, D., Kinney, A. L., \& Storchi-Bergmann, T. 1994, ApJ, 429, 582

Cardelli, J. A., Clayton, G. C., \& Mathis, J. S. 1989, ApJ, 345, 245

Chabrier, G. 2003, PASP, 115, 763

Charlot, S., \& Fall, S. M. 2000, ApJ, 539, 718

Dale, D. A., Helou, G., Contursi, A., Silbermann, N. A., \& Kolhatkar, S. 2001, ApJ, 549, 215

Dale, D. A., et al. 2005, ApJ, 633, 857 2007, ApJ, 655, 863

Devriendt, J. E. G., Guiderdoni, B., \& Sadat, R. 1999, A\&A, 350, 381

Draine, B. T., \& Li, A. 2007, ApJ, 657, 810

Faber, S. M., et al. 2007, ApJ, 665, 265

Fazio, G. G., et al. 2004, ApJS, 154, 10

Gil de Paz, A., et al. 2007, ApJS, 173, 185

Gordon, K. D., Clayton, G. C., Witt, A. N., \& Misselt, K. A. 2000, ApJ, 533, 236

Gordon, K. D., et al. 2007, PASP, submitted

Heckman, T. M., et al. 2005, ApJ, 619, L35

Helou, G., et al. 2004, ApJS, 154, 253

Hirashita, H., Buat, V., \& Inoue, A. K. 2003, A\&A, 410, 83

Iglesias-Páramo, J., et al. 2006, ApJS, 164, 38

Johnson, B. D., et al. 2006, ApJ, 644, L109

2007a, ApJS, submitted

2007b, ApJS, 173, 392

Jonsson, P. 2006, MNRAS, 372, 2

Kauffmann, G., et al. 2003a, MNRAS, 346, 1055

- 2003b, MNRAS, 341, 33

2007, ApJS, 173, 357

Kennicutt, R. C., Jr. 1998, ARA\&A, 36, 189

Kennicutt, R. C., Jr., et al. 2003, PASP, 115, 928

Kong, X., Charlot, S., Brinchmann, J., \& Fall, S. M. 2004, MNRAS, 349, 769

Kriek, M., et al. 2006, ApJ, 645, 44

Labbe, I., et al. 2007, ApJ, 665, 944

Li, Y., et al. 2007, ApJ, submitted (arXiv: 0706.3706v2)

Lonsdale, C. J., et al. 2003, PASP, 115, 897

MacArthur, L. A. 2005, ApJ, 623, 795

Mainzer, A. K., Eisenhardt, P., Wright, E. L., Liu, F.-C., Irace, W., Heinrichsen,

I., Cutri, R., \& Duval, V. 2005, Proc. SPIE, 5899, 262

Martin, D. C., et al. 2007, ApJS, 173, 415

Meurer, G. R., Heckman, T. M., \& Calzetti, D. 1999, ApJ, 521, 64

Mobasher, B., et al. 2004, ApJ, 600, L167

Morrissey, P., \& Seibert, M. 2007, ApJS, 173, 682

Moustakas, J., \& Kennicutt, R. C., Jr. 2006, ApJS, 164, 81

Murakami, H. 1998, Proc. SPIE, 3356, 471

Noeske, K. G., et al. 2007, ApJ, 660, L47

Panuzzo, P., Granato, G. L., Buat, V., Inoue, A. K., Silva, L., Iglesias-Páramo, J., \& Bressan, A. 2007, MNRAS, 375, 640

Papovich, C., \& Bell, E. F. 2002, ApJ, 579, L1

Pierini, D., Gordon, K. D., Witt, A. N., \& Madsen, G. J. 2004, ApJ, 617, 1022

Rieke, G. H., et al. 2004, ApJS, 154, 25

Salim, S., et al. 2007, ApJS, 173, 267

Schiminovich, D., et al. 2007, ApJS, 173, 315

Schlegel, D. J., Finkbeiner, D. P., \& Davis, M. 1998, ApJ, 500, 525

Schweizer, F., \& Seitzer, P. 1992, AJ, 104, 1039

Seibert, M., et al. 2005, ApJ, 619, L55

Silva, L., Granato, G. L., Bressan, A., \& Danese, L. 1998, ApJ, 509, 103

Smith, J. D. T., et al. 2007, ApJ, 656, 770

Stecher, T. P. 1965, ApJ, 142, 1683

Stoughton, C., et al. 2002, AJ, 123, 485

Strauss, M. A., et al. 2002, AJ, 124, 1810

Tinsley, B. M. 1968, ApJ, 151, 547

Tremonti, C. A., et al. 2004, ApJ, 613, 898

Treyer, M., et al. 2007, ApJS, 173, 256

Wang, B., \& Heckman, T. M. 1996, ApJ, 457, 645

Witt, A. N., \& Gordon, K. D. 2000, ApJ, 528, 799

Wuyts, S., et al. 2007, ApJ, 655, 51

Wyder, T. K., et al. 2007, ApJS, 173, 293 